ACQ:PPBD

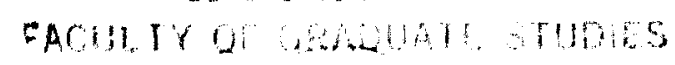

\title{
A COSMOLOGICAL FIELD THEORY
}

by

Steven Paul Starkovich

B.A., Oregun State University, 1976

M.S , University of Oregon, 1985

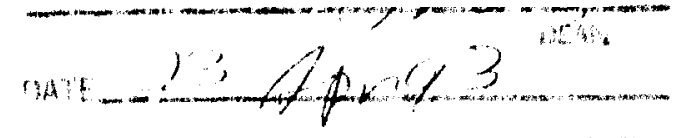

A Dissertation Sutmitted in Partial Fulfillment of the

Reyuiremen, for the Degree of

DOCTOR OF PHILOSOPHY

in the Department of Physics and Astronomy

We accept this dissertation as conforming to the required standard

Dr. F. I. Cooperstock, Supervisor

\begin{tabular}{c} 
Dr. C. E. Pieciotto, Departmental Member \\
\hline Dr. G. G. Miller, Cintsidt: Member \\
Dr. P. Hickson, External Examiner \\
STEVEN PAUL STARKOVICH, $19 \Omega 2$ \\
University of Victoria
\end{tabular}

All rights reserved. This dissertation may not be reproduced in whole or in part, by photocspying or other means, without the permission of the author. 
Supervisor: Dr. F. I. Cooperstock

\section{ABSTFiA $\underline{\text { CT }}$}

Ficld theory is used to describe the material content of the universe throughout its entire history, and an oscillating cosmological model without a singularity is presented. In our theory, the "cosmological fluid" is described by a classical scalar field tr. $\mathrm{At}$ undergoes a series of phase transitions over the lifetime of the universe. Each transition corresponds to a discontinuous change in the equation of statc of the field. In general, for an FRW universe and a given equation of state, we show that the field potential $V(\phi)$ may be derived from the solution of riccati's equation. The resulting expression for $V(\phi)$ includes parameters whose values are determined from the boundary conditions. In our theory, we employ the standard cosmological model and the fundamental Planck quantities to provide these boundary conditions. We thereby determine the scalar ficld Lagrangian for the entire history of the universe. The resulting cosmological model is free of any singularities, and includes an early inflationary epuch. Irflation arises in our theory as a consequence of the initial conditions. The theory describes a universe that is very cold at its minimum zadius, although $i$, heats rapidly during the initial inHationary era. This increase in the temperature of the scalar field during inflation is a direct consequence of applying classical thermodynamics under the assumed conditions for the early universe, and does not depend on the fine-tuning of free 
parameters. Inflation continues until a maximum possible physical temperature (the Planck temperature) is attained, at which point a phase transition occurs and the stan'ard model era begins. By relating the telaperature of the scalar field in our theory to the radiation temperature in the standard model universe, it is possible to establish a thermodynamic constraint on a more complete theory of matter for the early universe. Although, in principle, inflation occurs for any equation of state where $p<-(1 / 3) \rho$, we find that the initial equation of state must be $p \approx-\rho$ if the later epochs of the universe are to resemlle the standard model. In particular, we find that $H_{0}=33-44 \mathrm{~km} \mathrm{sec} \mathrm{c}^{-1} \mathrm{Mp}^{-1}$ is the value of the Hubble parameter at the current epoch that is least sensitive to the initial equation of state.

Examiners:

Dr. F. I. Cooperstock, Supervisor

Dr. F D. A. Hartwick, Departmental Mernber

Dr. C. E. Picciotto Departmental Menber

Dr. G.jG, Miller, Outsice Mernber, Department of Mathematics

Dr. P'. Hickson, External Examiner, Department of Physics, UBC 


\section{Contents}

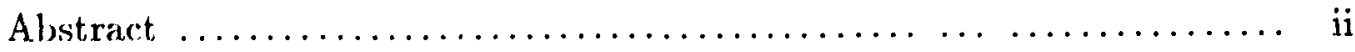

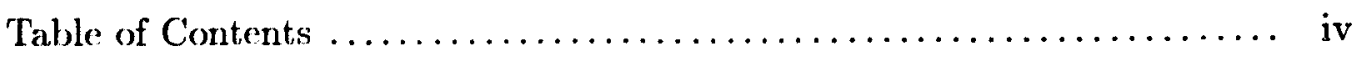

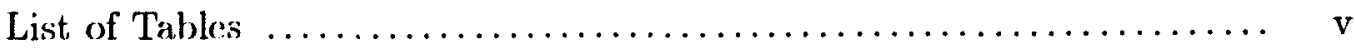

Acknowledgement $\ldots \ldots \ldots \ldots \ldots \ldots \ldots \ldots \ldots \ldots \ldots \ldots \ldots \ldots \ldots \ldots \ldots \ldots \ldots \ldots$

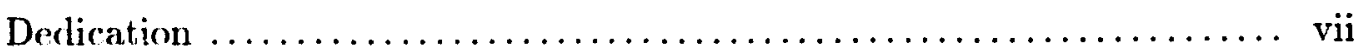

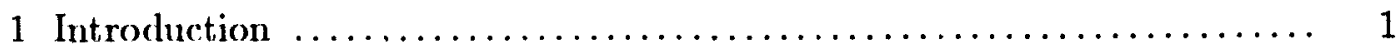

2 The Field Equations and Boundary Conditions $\ldots \ldots \ldots \ldots \ldots \ldots \ldots, 17$

3 The Determination of the Scalar Field Potential $\ldots \ldots \ldots \ldots \ldots \ldots \ldots \ldots$

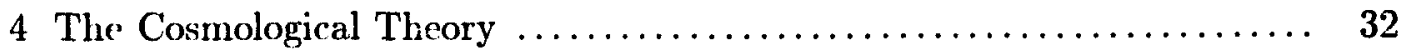

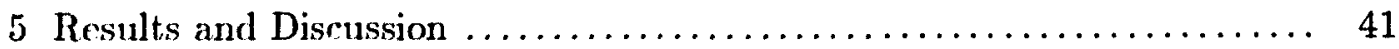

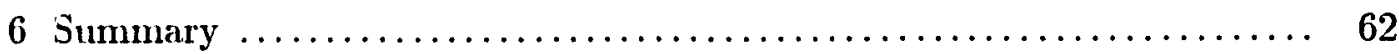

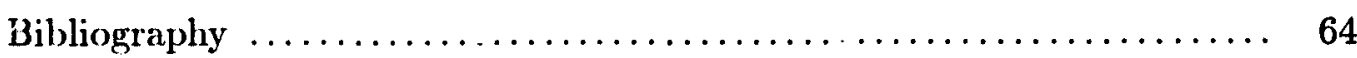

Appendix A: Natural Units $\ldots \ldots \ldots \ldots \ldots \ldots \ldots \ldots \ldots \ldots \ldots \ldots \ldots$

Appendix B: The Problems of the Standard Cosmological Model ...... 72






\section{List of Tables}

Table 1 - Solutions of Equations (4.11) for Varions Values of $\gamma_{p} \ldots \ldots \ldots \ldots 82$

Table 2 - Results for $\gamma_{p}=1.85 \times 10^{-3} \ldots \ldots \ldots \ldots \ldots \ldots \ldots \ldots \ldots \ldots \ldots \ldots$

Table 3 - Results for $\gamma_{p}=1.90 \times 10^{-3} \ldots \ldots \ldots \ldots \ldots \ldots \ldots \ldots \ldots \ldots$

Table 4 - Results for $\gamma_{p}=1.95 \times 10^{-3} \ldots \ldots \ldots \ldots \ldots \ldots \ldots \ldots \ldots$

Table 5 - Results for $\gamma_{p}=2.00 \times 10^{-3} \ldots \ldots \ldots \ldots \ldots \ldots \ldots \ldots \ldots \ldots \ldots$

Table 6 - Results for $\gamma_{p}=2.01 \times 10^{-3} \ldots \ldots \ldots \ldots \ldots \ldots \ldots \ldots \ldots$

Table 7 - Results for $\gamma_{p}=2.0153 \times 10^{-3} \ldots \ldots \ldots \ldots \ldots \ldots \ldots \ldots \ldots$

Table $\delta$ - Summary o ${ }^{\circ}$ Results for Various Values of $\gamma_{p} \ldots \ldots \ldots \ldots \ldots$ 90 


\section{ACKNOWIEDGEMENT}

I wish to express my thanks to Professor Fred Cooperstock for givang me the opportunity to pursue this rescarch. I am very grateful for his early onfidence and his continued support. In addition, I wish to express my appreciation for the financial assistance I received through the University of Victoria Fellowship. 


\section{Li.IICATION}

To

Ruth A. Williams 


\section{Chapter 1}

\section{Introduction}

Cosmology is the study of the origir. and evolution of the universe, and modnrn physical cosmology is inextricably bousd with gravitational theory. By one account (Bekenstein 1992), more than forty gravilational theories have been proposed, debated, and accepted or rejected since the second decade of the twentieth century. but it is the earliest of these theories - Einstein's theory of general relativity - that has been the most successful in describing the universe at large.

T'ie physical and historical significance of general relativity lies primarily in its fundamentally different view of space and time from that afforded by Newtonian theory. Einstein's theory asserts that gravity is a manifestation of the geometric structure of a four-dimensional spacetime manifold, and that this geometric structure is determined by the distribution of matter and energy within that spacetime. Unlike the othrr forces of nature, which are all formulated within an assumed underlying spacetime siructure, the phenomenon we call "gravity" is embodied within the rpacetime structure itself. According to Einstein's theory, gravitational attraction is manifested by the curvature of spacetime.

General relativity accomodates a broad range of assumptions regarding the 
structure and energy content of spaceime. With regard to cosmology, the most enpirically succ essful theory is that which, as it happens, follows from the simplest set of assumptions, namely, that the universe is homogeneous and isotropic, and that it is comprised of the familiar forms of matter and energy. Tlie assumptions of homogeneity and isotropy were motivated initially by considerations of simplicity and a preconception of the way the universe likely appears when virwed in the large. The resulting theory - the Friedman-Robertson-Walker (FRW) model has come to be known as the "standard cosmological model."

The compelling nature of the standard model arises frum its ability to predict several observed features of the universe in a consistent and comprehensive manner. These features include the expansion of the universe, the characteristics of the microwave background radiation, and the observed abundances of the light elements. Several important issues within the standard model remain unresolved, most notably those pertaining to a deternination of whether the universe is "open" or "closed" (a question that only makes sense once general rolativity is accepted as the underlying theory of gravity) and the details of galaxy formation. However, during the past two decades, most of the critici'm of the standard model has stemmed from what physicists have deemed to be an unacceptalsle dependence on a precise adjustment (a "fi: e-tuning") of initial conditions necessary to yicld the universe that we observe. These criticisms are usually expressed is the well- 
known horizon, smoo'hness, and flatness "problems." The flatuess problem is the need to f,recisely adjust the conditions of the early universe so that it resembles a flat universe to a very high precision. The horizon and smoothness problems raise the issue as to why causally disconnecter. regiors of the standard-model universe are so similar in their physical charpateristics. These issues are described in more detail in Appendir B.

The past two decades also have borne witness to $\mathrm{m}$ ajor advances in theoretical particle physiss. The accepted model of particle physics, articulated in the language of quantum field theory, accomodates the existing experimental evidence of particle interactions. Nonetreless, there remains substantial theoretical discomfort with the number of free parameters within the model for which there are no compelling restrictions. In addition, there is an aesthetic motivation for ever-higher degrees of unification of the forces of nature. The effort to extrapolate quantum field theory to energy realms exceeding those attainable in the laboratory has led logically to a symbiosis between particle physics and cosmology. Consequently, the universe - particularly the early universe - nas become a "iaboratory" in which new field theories of matter may be tested for their cosmological consequences. Of course, the present low-energy observable universe (with photons at an $€$ nergy of approximatcly $10^{-13} \mathrm{GeV}$ ) can provide a very-high-energy particle physics laboratory (with Planck-scale encrgies of $10^{+19} \mathrm{GeV}$ ) only if an 
assumption is mide as to an inderlying theory of cosmological erolution. There fore, to a great extent, the viability of any given unified ther $y$ of particies and fieids has come to depend upon the valulity of the standard theory of cosmology.

The most notew orthy product of the symbiosic between field theory and cos mology is the inflationary universe theory that has served to alleviate the fitctuning problems of the standard model. Tc lay, the assumption that there exists an inflationary epoch in the early universe -... whercin the scale factor of the uni verse acceierates - is well-accepted. The recent literature coltaius numerous comprehensive reviews of modern cosmology and inflation theories, anong them being Börner 1988, Brandenberger 1985, Collins et al. 1989, Bolb and Turure 1990, Linde 1990, and Olive 1990. The discussion in this Introduction draws upon these reviews.

The field theory formalism for the description of matter and the forces of nature has a long history (Pickering 1984). The objective of a field theory (whether quantum or classical) is the specification of a locally-invariant Lagrangian that is comprised of the various matter and gauge fields appropriate to the problem under consideration, and that yields equa : ons whose solutions describe the phenomena of interest. For our purposes here, it is useful to outline the basic notions le. hind the most generally-accepted contemporary cosmological theories so that we might better understand the relationship between the ficld theoretic description 
of matter and cosmological inflation.

In these theories, the Lagrangian - which may include a variety of fields corresponding to different fundamental particles - typically includes a scalar field whose potential em. . lies the energy content of the vacuum. The pre-inflationary uriverse is presumed to be radiation-dominated and at a very high temperature, so that as the universe emerges from a singularity, the scale factor $R \sim t^{1 / 2}$ while the temperature $T \sim 1 / R$. When the temperature falls below a critical value, the vacunum energy density dominates the radiation density, and the vacuun-dominated universe (with the equation of state $p=-\rho$ ) inflates beyond the particle horizon. The result is a causally-connected observable universe. Inflation ends when the symmetry of the scalar field potent:-1 is broken, either by a tunnelling process as in the "old inflation" theory, or by a "slow-rcllover" process as in "new inflation." The symmetry breaking serves to redefine, or restructure, the ground state (the vaculum) of the theory. The radiation, having croled exponentially during inflation, must then be reheated.

Although se wal contemporary inflation theories have offered solutions to the problems of the standard model, fundamental problems still remain with most inflation models. Perhaps the most serious problem is the fact that a generallyaccepted field theory of matter for the early universe does not exist. The simplest grand unified theories have been shown to be unsatisfactory from both a particle 
p. vsies and cosmological perspective, and most other mociels are still extremely speculative. Further, in those circumstances where a particular theory of matter is being investigated for its cosmological consequences, it is usually necessary to finely tune one or more free parameters in the model in order for the cud result to be a universe resembling the one that we observe. The fine-tuning problems are most apparent with regard to the specification of the details of the transition between the inflation era and the standard model an issue that has plagued inflation models since their inception. Thus, the fine-tuning problems of the standard cosmological model have, to some extent, been recast as finc-tuning problems within the field theory that accounts for inflation. Finally, in many inflation models, a scalar field is in:iuded in the theory for the sole purpose of generating inflation, and is not otherwise integrated into a comprehensive theory of matter.

It is against this background that we now describe the motivation for the present work. We wish to develop a cosmological field theory that unifies the early and late epochs of the universe within a single theoretical framework that employs field theory for the description of the material content of the universe. While quantum field theory forms the basis of other contemporary morlels of the early universe, this dissertation describes a cosmological theory rooted in the long tradition of classical field theory. While the standard model of particle physics is the foundation upon which all other cosmological models are constructed, the 
theory of matter we employ in this dissertation envisions the material content of the universe as a continuum.

The ramifications of our theory will become clear as we proceed through this dissertation. Even though the fundamental notions about the nature of matter which underlie the present work differ substantially from the ideas underlying other contemporary theories of the early universe, there are nonetheless some common themes. In the remainder of this Introduction we shall anticipate some of our results and make a few comparisuns between the two approaches. It shall then be easier to appreciate une advantages, as well as the disadvantages, that our theory holds over other models.

An important concept that bears an immediate relevance to the present work is an idea due to Markov (1982) who suggested that, in much the same way that there exists a limiting physical velocity, there might exist a limiting density, and limits on other physical quantities as well. Markov identifies the Planck density as a naturad limiting density. Contemporary inflation models provide for an early universe that emerges from a singularity into a radiation-ciominated state, then becomes vacuum-dominated, and then evolves to the radiation-dominated epoch that marks the beginning of the standard model era. If, instead, the universe begins its expansion in a "vacuum-like" state, then general relativistic cosmology permits the initial conditions to be such that the Hubble parameter vanishes when 
the density is at its limiting value. If the energy density is positive, the Einsicin equations then require the universe to be closed.

Therefore, these two considerations -- an initial vacuun-like equatio"' of state in the early uriverse and a limiting physical density load logically to a closed universe without an initial singularity. The presence of an initial singularity signifies a breakdown of physics at the beginning of the universe. It is reasonable to require that singularity avoidance be a criterion in any plysical theory, and the use of a vacuum-like equation of state ;o avoid a singularity in the carly universe has a long history that extends at least as far back as Gliner (1966). More recently, a singularity-free cosmological model with an carly inflationary cpoch was proposed by Israelit and Rosen (1989). In their model, which does not employ field theory for the description of the material content of the universe, they postulate that the early universe is comprised of a material substance (they refor to it as "prematter") whose equation of state is initially the same as that for the vacuum. They construct a continuous function for the equation of state such that, as the universe evolves, the inflationary prematter epoch is followed by the radiation- and matter-dominated epochs of the standard model. They do not, however, provide a theory of matter that accounts for this behavior.

Cosmological models which do not use field theory lack tive fundannental theoretical underpinnings that field theory provides for the description of matter. 
On the: other hand, as we have already noted, a surcessful quantum field theory for matter in the early universe has yet to ho discovered. In tais dissertation, we develo: a cosmological theory utilizing a single, classical scalar field to represent the materi: ${ }^{\prime}$ content of the universe. This approach is a logical extension of that used by Coope:stork and Rosen (1989) to construct static particle solutions to the field equations of a classical field theory. In a sense, changing the boundary conditions and the independent variable cause the static, scalar field solutions of a particle theory to become cosmological models wherein the material content of the universe is a scalar field continuum.

The theory we present goes beyond a consideration of the early universe alone, and describes the general behavior of the universe throughout its entire history. In addition, we consider a broader range of initial conditions than was considered in the work of Israelit and Rosen, although many of the physical results they obtained are qualitatively similar to the res'lts we shall eventually obtain from our theory.

The scalar field in our theory - the "cosmological fluid" - represents the total material content of the universe and we postulate that the field may attain various equations of state in order that various cosmological epochs may be analyzed. Accordingly, the "radiation" and "matter" epochs are those eras in the history of the universe where the scalar field attains the equation of state 
$p=(1 / 3) \rho$ and $p=0$, respectivaly. We shall use the term "prematter" (from Israelit and Rosen) to describe the physical state of the scalar field during the inflation era where the equation of state approaches $p=--\rho$.

Discontinuous changes in the equation of state of the scalar ficld in our thery denote the occurrence of phase transitions. These transitions occur at critical ficld values, and our theory permits the determination of these values. Our use of the phase transition concept removes the arbitrariness associated with constructing a functional form for the equation of state, particularly for the transitions between the phases. However, we must make it very clear that our use the phase transition concept is much different from that which appears in the literature on field theories of the early universe; so different, in fact, that only some terminology is similar. Vacuum phase transitions are a staple of most contemporary cosmological theories, and they are manifested by a symmetry-brea'ing of a scalar field potential in accordance with the generalized theory of phase transitions due to Landau (Landau and Lifshitz 1980).

These other theories include the scalar field as only one of several compon its of the cosmological fluid. By contrast, in our theory, the entire material cont,nt is embodied within a single component - the scalar field -... whose value evolves smoothly over time. A phase transiticn is then nothing more than a change in the equation of state of the field. The transition take' place at a particular field 
value which we refer to here as a "critical value." There is no change in the nature of the potential and no sudden change in the field value to mark the occurrence of the transition … this latter feature being the central idea behind the Landau theory. Consecuuently, our scalar field does not play the role of an order parameter in a vacuum phase transition as it does in other theories.

From a practical point of view, our theory should be able to speak to the important observable features of the universe. Among these features is the temperature of the microwave background radiation. The application of the first and second laws of thermodynamics to our theory shows that the temperature of the scalar field must increase during the inflationary era. This is a consequence of simplifying the assumptions about the early universe - that is, by eliminating a pre-inflationary radiation era - and of embodving the material content solely within the scalar field. Thus, it is possible to reconcile inflation and "heating" by appealing to the laws of thermodynamics within the context of the assumptions in our theory. Consequently, inflation and heating become complementary phenomena, unlike in other theories where a "reheating" of a separate radiative component must be introduced after inflation to compensate for the exponential cooling of the radiation during inflation. Of course, the actval universe contains a radiative component, and later in this paper we discuss how the temperature of the scalar field in our theory can be related to a radiation temperature in the 
observed universe. The result of this discussion is what we shall refer to as a "thermodynamic constraint," on a more complete theory of matter for the early universe.

In further accordance with the idea that there are finite limits to physical quantities, we assume that there exists a maximum possible physical temperature. This limit is taken to be the Planck temperature $\left(T_{p l}\right)$, whose vaiue may be obtained by setting $\tau_{p l}=m_{p l}$, where, in natural units (see Appendix A), the Boltzmann constant equals one. lia our theory, inflation ends by a basic physical consideration - the attainment of this maximum possible temperature and not means of a finely-tuned mechanisin as required in other field theories of the early universe. When this maximum temperature is attained, a transition from a vacuum-like equation of state to a radiative equation of state occurs. At this transition, inflation ends and the era described by the standard model begins.

The mission of other field theories of the early universe is completed upon the transition from an inflationary epoch to the beginning of the standard model. In contrast, our theory unifies the early and late epochs withia the same theoretical framework while still providing for a later universe that follows the general outline of the radiation and matter epochs of the standard model. As is the case during the early inflationary epoch, the material content of the universe during the later epochs also is described by the same scalar field, but now with a different equation 
of state. Because the universe in our theory is closed, it will eventuany reach a maximum size, contract to a si:ale of the order of the Planck length, and then re-expand, retracing its steps cyclically (that is, the scale ficto: of the universe will continue to "oscillate" between its ranimum and maximum values). We adopt the familiar idealization that the universe is homogeneous and isotropic, and this assumption is important with regard to singularity-avoidance upon the contraction of the universe. We discuss this issue 1 ear the end of this dissertation.

There is at least one other imfortant difference between our theory and most others, and it is a difference of approach toward solving the field equation :. In ther models, a particular scalar field potential is moivivated by some independent consideration and then tested for its cosmological consequences. In contrast, our approach is to determine the scaiar field potential which vields reasonable cosmological results throughout the entire history of the universe. In other words, we shall appeal to the standard cosmological model in order to establish the potential function corresponding to that behavior. Toward this end, we show that for an FRW universe and a given equation of state, the field potential may be derived from the solution of Riccati's eq ation. Alternatively, our development may be altered slightly so that the equation of state may be determined if the potential is known. This could be of interest in those studies that examine the cosmological consequences of a particular potential. 
We now summarize the important features of the cosmological field theory presented in this dissertation.

1) The universe is closed and oscillates between a minimum and maximum size. Arguments against oscillating models that are founded upon completely different theoretical premises from those in the present work have no bearing on this result (for example, see Guth and Sher 1983);

2) The early universe includes an inflationary epoch that arises as a consequence of the imposed boundary conditions which, in turn, are expressed in terms of the fundamental constants of nature (the Planck quantities). Inflation is independent of the existence of an earlier radiation-dominated universe;

3) The universe is initially very cold, but its temperature increcses rapidly. This increase in the temperature of the scalar field is complementary with inflation, it follows from basic thermodynamic considerations, and it does not require a finetuning of free parameters;

4) Relating the temperature of the scalar field to a radiative temperature in the standard model yields a thermodynamic constraint on a more complete theory of matter that may be superimposed on our model while retaining our general results; 
5) The attainment of a physical state (a maximum possible physical temperature) marks the end of inflation. There is no need to construct a finely tuned mechanism for making a transition from inflation to the staridard model;

6) The material content of the universe is described by a field theory that is relevant for the entire history of the universe, rather than only for an early epoch. The scalar field potential (and thereby the Lagrangian function) that describes the evolution of the universe throughout its entire history is established; and

7) After utilizing all the boundary conditions, the only remaining free parameter in the entire theory is the initial equation of state. It is found that without excessive fine-tuning - the initial equation of state must be $p \approx-\rho$ if the later epochs are to resemble the standard model. We are able to predict the value of the Hubble parameter at the present epoin that is least sensitive to the choice of the initial equation of state.

In addition, the theory also perm:ts the determination of the critical values of the scalar field where the phase transitions occur. Finally, we discover that the scalar field possesses a negative specific heat during the prematter phase (that is, its temperature increases while its density decreases).

In Chapter 2 we develop the basic equations and specify the boundary conditions. Most of the content of Chapter 2 is well-known and may be found in many 
other sources in the literature, including the review monographs cited carlier. W: include this material here tor the sake of completeness. In Chapter 3, we derivo the differential equation - whose form is that of Riccati's equation from which we then determine the field potential. In Chapter 4 we develop the cosmological theory in detail, and in Chapter 5 we present the cosmological results and offer further discussion of the theory. 


\section{Chapter 2}

\section{The Field Equations and Boundary Conditions}

The Einstein equations of general relativity are

$$
G_{i}^{k}=8 \pi T_{i}^{k}
$$

where $G_{i}^{k}$ and $T_{i}^{k}$ are the Einstein and energy-momentum tensors, respectively, and the indices $i$ and $k$ take on the values $0,1,2$, and 3 . We adopt the natural system of units $(\hbar=c=1)$ so that the gravitational constant $G=1 / m_{p l}^{2}$, where $m_{p l}$ is the Planck mass. The equations are further simplified if all physical quantities, including the gravitational constant, are measured in Planck units (see Appendix A).

Because we assume the universe to be homogeneous and isotropic, we adopt the Friedmann-Robertson-Walker (FRW) spacetime whose interval is given by

$$
d s^{2}=d t^{2}-R^{2}\left[\frac{d r^{2}}{1-k r^{2}}+r^{2}\left(d \theta^{2}+\sin ^{2} \theta d \phi^{2}\right)\right]
$$

where, generally, $d s^{2}=g_{i k} d x^{i} d x^{k}$ and $g_{i k}$ is the metric tensor.

In equation $(2 .:) t$ is the co-moving (proper) time as measured in Planc: times, $r$ is the dimensionless co-moving coordinate distance, and $R$ is the scale 
factor of the universe. The parameter $k$ denotes the spatial curvature and takes on the values $k=+1,-1$ or 0 for positive, negative, or zero curvature, respertively. If $k=+1, R$ is the radius of the universe in Plancl lengths; otherwise $R$ is only a measure of the relative proper separation of given noints at differrut times. For the rcasons est blished in C. apter 1 , we shall be considering a closed $(k=+1)$ model in this dissertation.

The energy-momentum tensor in equation (2.1) is that of the matter producing the gravitational field. In order to describe the material content of the universe within the field theory formalism, it is sufficient to specify the Lagrangian of the theory. The Lagrangian in our theory is that of a real scalar ficld $\phi$, and may be written in the standard form

$$
L=\frac{1}{2}\left(\partial_{i} \phi\right)\left(\partial^{i} \phi\right)-V(\phi)
$$

where $V(\phi)$ is the potential.

There are two expressions for $T_{i}^{k}$ :

$$
T_{i}^{k}=\left(\partial_{i} \phi\right)\left(\partial^{k} \phi\right)-\delta_{i}^{k} L
$$

which follows from equation (2.3) and the definition of $T_{i}^{k}$ from field theory; and

$$
T_{i}^{k}=(\rho+p) u_{i} u^{k}-p \delta_{i}^{k}
$$

which applies to any macroscopic perfect fluid (Landau and Lifshitz 1975). In these equations, $\rho$ is the density, $p$ is the pressure, and $u^{i}=\left(u^{\prime \prime}, u^{\alpha}\right)$ is the four- 
velocity of the cosmological fluid, where $\alpha$ takes on the values 1,2 , and 3 . The spatial components, $u^{\alpha}$, vənish under the constraints of homogeneity and isotropy. Comparison of these expressions for $T_{i}^{k}$ yields expressions for $\rho$ and $p$ in terms of the field quantities:

$$
\begin{aligned}
& \rho=\frac{1}{2} \dot{\phi}^{2}+V(\phi) \\
& p=\frac{1}{2} \dot{\phi}^{2}-V(\phi) .
\end{aligned}
$$

An additional term $\left(\tau_{i}^{k}\right)$ could have been included in equation (2.5) for the purpose of representing a contribution to the energy-momentum tensor from dissipative processes within the cosmological fluid. The most general form for $\tau_{i}^{k}$, given by

$$
\tau_{i}^{k}=\eta\left[u_{; i}^{k}+u_{i}^{; k}-u^{l} u_{i} u_{; l}^{k}-u^{l} u^{k} u_{i ; l}\right]+\left(\zeta-\frac{2}{3} \eta\right)\left(\delta_{i}^{k}-u_{i} u^{k}\right) u_{; l}^{l}
$$

(Landau and Lifshitz 1987), would be reduced to

$$
\tau_{i}^{k}=\zeta\left(\delta_{i}^{k}-u_{i} u^{k}\right) u_{; l}^{l}
$$

under the coustraints of homogeneity and isotropy. In these equations, $\eta$ is a sh.ar viscosity coefficient and $\zeta$ is a bulk viscosity coefficient for the cosmological fluid. In the FRW metric $u_{; l}^{l}=3 H$ where $H \equiv \dot{R} / R$ is the Hubble parameter. Thus, the whole effect of $\tau_{i}^{k}$ in our theory would be to replace the pressure $p$ with the quantity $\vec{i}^{*} \equiv p-3 H \zeta$ (Weinberg 1972). In this paper, we assume the 
cosmological fluid is well-approximated by a perfect fluid, and therefore $\tau_{i}^{k}=0$. As a result, no entropy production can be forthcoming from the dynamics of the scalar fieid.

Evaluating the 0-0 and $\alpha-\alpha$ components of equation (2.1), and using equations (2.2) and (2.5), we obtain two equations which describe the time evolution of a homogeneous, isotropic universe:

$$
\left(\frac{\dot{R}}{R}\right)^{2}+\frac{k}{R^{2}}=\frac{8 \pi \rho}{3}
$$

and

$$
\ddot{R}+\frac{4 \pi}{3}(3 \gamma-2) \rho R=0
$$

We have defined the parameter $\gamma$ as

$$
\gamma \equiv \frac{p}{\rho}+1=\frac{2 \dot{\phi}^{2}}{\dot{\phi}^{2}+2 V}
$$

so that

$$
V=\rho \frac{(2-\gamma)}{2}
$$

where we have used equations (2.6) and (2.7). If $\gamma=4 / 3$ the universe is radiationdominated, while if $\gamma=1$ the universe is matter-dominated $(p=0)$. The case: of $\gamma=0$ corresponds to the vacuum equation of strite $(p=-\rho)$. From equation (2.11), we see that inflation $(\ddot{R}>0)$ arises for any $\gamma<2 / 3$.

Equations (2.10) and (2.11) are related to each other by the conservatio"1 of energy equation which is found from the $i=0$ component of the covariant 
conservation laws $T_{i ; k}^{k}=0$. The result may be written in at least three equivalent forms:

$$
\ddot{\phi}+3 H \dot{\phi}+\frac{\partial V}{\partial \phi}=0
$$

(which can also be obtained by varying the Lagrangian with respeci to $\phi$ ),

$$
\dot{\rho}+3 H \gamma \rho=0
$$

or

$$
\frac{d}{d R}\left(\rho R^{3}\right)=-3 p R^{2}
$$

The two independent field equations we shall solve are equation (2.13) and

$$
\ddot{R}+\kappa\left(\dot{\phi}^{2}-V\right) R=0, \quad \kappa \equiv \frac{8 \pi}{3}
$$

which follows from equations (2.6), (2.11), and (2.12).

The first and second laws of thermodynamics combine to give the Gibbs relation

$$
T d S=d E+p d\left(2 \pi^{2} R^{3}\right)
$$

where $S$ and $E$ are the total entropy and energy, respectively, and $2 \pi^{2} R^{3}$ is the volume of a $k=+1$ universe of radius $R$. Letting $s$ and $\rho$ be the entropy and energy densities, and applying the integrability conditions of equation (2.17), we obtain (Weir.berg 1972)

$$
s=\frac{1}{T}(\rho+p)=\frac{\gamma \rho}{T}
$$


where we have used equation (2.12a).

Differentiating equation (2.17) with respect to $R$, and using equation (2.15), we obtain an equation that reflects the perfect fluid assumption:

$$
T \frac{d}{d t}\left(s R^{3}\right)=0
$$

Carrying out the differentiation in equation (2.19), and using equations (2.14) and (2.18), we obtain

$$
\dot{\gamma}-\gamma\left(\frac{\dot{T}}{T}\right)-3 H \gamma(\gamma-1)=0
$$

Therefore, once the field equations are solved, the temperature evolution of the cosmological fluid may be determined from equations (2.12) and (2.20). The evolution of the density and pressure may also be found from equations (2.12).

In order to establish the initial conditions for the field equations, we assume that the maximum attainable density in any physical system is the Planck density (Markov 1982). When this constraint is applied in conjunction with the initial condition $\dot{R}(0)=0$, the complete set of initial conditions becomes

$$
\begin{gathered}
\rho(0)=1 \\
\dot{\rho}(0)=0 \\
R(0)=\left(\frac{1}{\kappa}\right)^{\frac{1}{2}} \\
\dot{R}(0)=0
\end{gathered}
$$


where $\rho$ is given in terms of the field quantities by equation (2.6), and where we have used equation (2.10) with $k=+1$.

In Chapter 4 it shall become apparent that we need two additional boundary conditions in order to complete the cosmological theory. These boundary conditions are established by considering the radiation epoch of the standar $d$ cosmological model. At the end of the radiation epoch, the radiation temperature is approximately $2900^{\circ} \mathrm{K}$ (Kolb and Turner 1990). This corresponds to a radiation energy density at decoupling of

$$
\rho_{d e c}=\frac{A \pi^{2}}{15}
$$

Planck densities, where $A=1.7539 \times 10^{-115}$. As one of the boundary conditions, we shall assume that the energy density in equation (2.22a) equals the energy density of the scalar field at the corresponding time in our theory.

In our theory, the radiation epoch begins when the temperature has attained a maximum value. This is a consequence of equation (2.20) and a feature of our model which will be specified later in this paper after a more general development in Chapter 3 ; namely that $\gamma$ will be a specified constant for a given epoch. Then, since $\gamma<2 / 3$ during the inflation era while $\gamma=4 / 3$ during the radiation era, equation (2.20) shows that $\dot{T}>0$ during inflation and $\dot{T}<0$ during the radiation era, that is, $T$ reaches a maximum at the beginning of the standard model era. 
For the purpose of establishing the value of this maximum temperature, we assume that the energy density at the beginning of the radiation era of the standard model is given by

$$
\rho_{r, i}=\frac{\pi^{2}}{15} T_{r, i}^{4} \equiv \frac{\pi^{2}}{15}
$$

where the temperature has been scaled in such a way that $T_{r, i}=1$. This natural scaling of the temperature, in conjunction with equation $(2.22 \mathrm{a})$, yields the Planck temperature

$$
T_{p l} \equiv T_{r, i}=1.42 \times 10^{32}{ }^{\circ} \mathrm{K}
$$

This is the same temperature that one would obtain from setting $T_{p l}=m_{p l}$. We shall assume that the energy density of the scalar field at the end of the inflation era in our theory is given by equation $(2.22 \mathrm{~b})$. This is the second boundary condition we seek.

These two boundary conditions allow us to complete our cosmological theory, while appealing to the standard model for the determination of two free parameters. We should note that the values for $\rho$ in equations (2.22) are calculated under the simplifying assumption that only photons contribute to the energy density during the radiation era of the standard moael. The qualitative behavior of our model would not be affected were we to include other relativistic species when specifying the vaiues in equations (2.22).

Equations (2.13) and (2.16) are a system of two equations in three unknowns, 
and may be solved if either $\gamma$ or $V$ is specified. The approach in this paper will be to specify an equation of state in terms of the field $\phi$. The field equations are then solved and, as we show in Chapter 3, the scalar field potential is determined. We thereby obtain the Lagrangian of the field theory for the entire history of the universe. By speciaying $\gamma$, our approach more closely resembles that used in standard cosmology than the approach used in quantum field theories of the early universe. In the latter, the potential $V(\phi)$ is postulated and then tested for its cosmological consequences. 


\section{Chapter 3}

\section{The Determination of the Scalar Field Potential}

If $\phi$ is a constant, equations (2.12) - (2.16) show how the behavior of the scale factor depends on the value of $\partial V / \partial \phi$. In particular, if $\partial V / \partial \phi=0$ when $\dot{\phi}=0$, the scale factor undergoes eternal exponential inflation, and $R(t) \sim \exp \left(\chi^{t}\right)$ for all time, where $\chi=(8 \pi \rho / 3)^{1 / 2}$ and the density is constant. On the other hand, if $\partial V / \partial \phi \neq 0$ for constant $\phi$, then $\dot{\phi}$ evolves away from zero and eternal inflation is avoided.

In the remainder of this dissertation, we consider only the case of $\dot{\phi} \neq 0$ or, equivalently, $\gamma \neq 0$. We proceed on the assumption that an effective equation of state $\gamma=\gamma(\phi)$ is specified. In this Chapter, the field equations are transformed into equations for $\ln V$ and $\ln R$ as functions of $\phi$, and these new equations are, in turn, combined to yield a Riccati equation whose solution permits us to find $V(\phi)$

Equation (2.12a) is differentiated with respect to time and rearranged to yield

$$
\ddot{\phi}=\frac{\dot{\phi}}{2 V}\left[\frac{\dot{\gamma} \dot{\phi}^{2}}{\gamma^{2}}+\dot{V}\right] .
$$


Equation (2.13) is transformed using equation (2.12a), equation (3.1), and the Hubble parameter $(H)$ to obtain

$$
\frac{d \ln R}{d \phi}=-\frac{1}{3}\left[\frac{1}{\gamma} \frac{d \ln V}{d \phi}+\frac{1}{2-\gamma} \frac{d \ln \gamma}{d \phi}\right]
$$

In order to transform equation (2.16), we first differentiate the Hubble parameter with respect to time:

$$
\frac{\ddot{R}}{R}=\dot{H}+H^{2}=\ddot{\phi} \frac{d \ln R}{d \phi}+\dot{\phi}^{2} \frac{d^{2} \ln R}{d \phi^{2}}+\dot{\phi}^{2}\left(\frac{d \ln R}{d \phi}\right)^{2} .
$$

Equations (2.12a), (3.1) and (3.3) are combined to yield

$$
\frac{\ddot{R}}{R \dot{\phi}^{2}}=\left[\frac{1}{2-\gamma} \frac{d \ln \gamma}{d \phi}+\frac{1}{2} \frac{d \ln V}{d \phi}\right] \frac{d \ln R}{d \phi}+\frac{d^{2} \ln R}{d \phi^{2}}+\left(\frac{d \ln R}{d \phi}\right)^{2}
$$

an expression that arises only from the definitions of $H$ and $\gamma$.

In addition, equations (2.12) and (2.16) are combined to yield

$$
\frac{\ddot{R}}{R \dot{\phi}^{2}}=\frac{\kappa}{2 \gamma}(2-3 \gamma) \text {. }
$$

Comparison of equations (3.4) and (3.5) yields

$$
\left[\frac{1}{2-\gamma} \frac{d \ln \gamma}{d \phi}+\frac{1}{2} \frac{d \ln V}{d \phi}\right] \frac{d \ln R}{d \phi}+\frac{d^{2} \ln R}{d \phi^{2}}+\left(\frac{d \ln R}{d \phi}\right)^{2}=\frac{\kappa}{2 \gamma}(2-3 \gamma)
$$

Equations (2.13) and (2.16) have thus been transformed into equations (3.2) and (3.6). In a similar way, equation (2.20) is transformed into

$$
\frac{d \ln \gamma}{d \phi}-\frac{d \ln T}{d \phi}-3(\gamma-1) \frac{d \ln R}{d \phi}=0 .
$$


Differentiating equation (3.2) with respect to $\phi$ yields

$$
\frac{d^{2} \ln R}{d \phi^{2}}=-\frac{1}{3}\left[\frac{1}{\gamma} \frac{d^{2} \ln V}{d \phi^{2}}-\frac{1}{\gamma} \frac{d \ln \gamma}{d \phi} \frac{d \ln V}{d \phi}+\frac{1}{2-\gamma} \frac{d^{2} \ln \gamma}{d \phi^{\circ}}+\frac{\gamma}{(2-\gamma)^{2}}\left(\frac{d \ln \gamma}{d \phi}\right)^{2}\right]
$$

Substituting equations (3.2) and (3.8) into equation (3.0́) and collecting terms yields

$$
\frac{d u}{d \phi}+a(\phi) u+b(\phi) u^{2}+c(\phi)=0
$$

where $u \equiv d \ln V / d \phi$,

$$
\begin{gathered}
a(\phi) \equiv \frac{9 \gamma-10}{6(2-\gamma)} \frac{d \ln \gamma}{d \phi} \\
b(\phi) \equiv \frac{3 \gamma-2}{6 \gamma}
\end{gathered}
$$

and

$$
c(\phi) \equiv \frac{\gamma}{2-\gamma} \frac{d^{2} \ln \gamma}{d \phi^{2}}+\frac{\gamma(3 \gamma+2)}{3(2-\gamma)^{2}}\left(\frac{d \ln \gamma}{d \phi}\right)^{2}+\frac{3 \kappa}{2}(2-3 \gamma)
$$

Equation (3.9) is of the general form of Riccati's equation for $u$ and is solved by converting it to a second-order, linear, homogeneous differential equation (Davis 1962). A function $w(\phi)$ may be defined such that

$$
u(\phi) \equiv \frac{1}{b(\phi)} \frac{w^{\prime}(\phi)}{w(\phi)}
$$

where the' denotes differentiation with respect to $\phi$.

Substituting equation (3.11) into equation (3.9) yields

$$
w^{\prime \prime}+\left[a(\phi)-\frac{b^{\prime}(\dot{\phi})}{b(\phi)}\right] w^{\prime}+[b(\phi) c(\phi)] w=0
$$


The task of solving the field equations has thus been reduced to solving equation (3.12). The most important special case of equation (3.12), and the one which we shall consider for the remainder of this dissertation, is for $\gamma$ equal to a constant. In this: circumstance, equations (3.10) become

$$
a=0 ; \quad b=(3 \gamma-2) / 6 \gamma ; \quad c=3 \kappa(2-3 \gamma) / 2
$$

Substituting equation (3.13) into equation (3.12), we obtain a differential equation for $w(\phi)$

$$
w^{\prime \prime}-\alpha^{2} w=0
$$

where

$$
\alpha \equiv 3 b(\kappa \gamma)^{1 / 2}
$$

Equation (3.1.ta) has the general solution

$$
w(\phi)=\beta_{1} e^{-\alpha \phi}+\beta_{2} e^{\alpha \phi}
$$

where $\beta_{1}$ and $\beta_{2}$ are constants of integration.

Equation (3.11) then yields

$$
\frac{d \ln V}{d \phi}=-\frac{\alpha}{b}\left[\frac{1-\beta e^{2 \alpha \phi}}{1+\beta e^{2 \alpha \phi}}\right]
$$

which, upon integration, yields

$$
V(\phi)=C\left(e^{-\alpha \phi}+\beta e^{\alpha \phi}\right)^{1 / b},
$$


where $\beta \equiv \beta_{2} / \beta_{1}$ and $C$ is a constant of integration.

Equation (3.17) is the gener: solution for the scalar field potential under the condition that $\gamma$ is a nonvanishing constant. We emphasize again that, at this stagc of the development, no other boundary conditions have been imposed. In particular, this result is applicable to all inflation models which can accomodate. this condition on $\gamma$, and this includes a wide range of models currently in the literature. The constants $\beta$ and $C$ in equation (3.17) are determined from whatever additional boundary conditions are imposed in conjunction with a particular application of this general result.

Finally, equations (3.2) and (3.7) become

$$
\frac{d \ln R}{d \phi}=-\frac{1}{3 \gamma} \frac{d \ln V}{d \phi}
$$

and

$$
\frac{d \ln T}{d \phi}=\left(\frac{\gamma-1}{\gamma}\right) \frac{d \ln V}{d \phi}
$$

and equation $(2.12 \mathrm{a})$ yields

$$
\dot{\phi}= \pm\left(\frac{2 \gamma}{2-\gamma}\right)^{1,2} V^{1 / 2}(\phi)
$$

The choice of either the plus or minus sign in equation (3.20) will depend upon other conditions imposed on the problem.

It should be noted that it is possible to combine the field equations in a different way and still solve for the temporal evolution of the scalar field potential. 
One such approach was developed by Ellis and Madsen (1991) where an explicit dependence on the Hubble parameter is retained in their equations. Jur method pursues a different line of development that eventually leads to an equation of the form of Riccati's equation from which we can then determine an expression for $V(\phi)$. A cosmological theory may now be constructed by applying these results to consecutive cosmological epochs, each with its own characteristic value of $\gamma$. The construction of such a theory is the subject of Chapter 4 . 


\section{Chapter 4}

\section{The Cosmoloyical Theory}

here are many possible applications of equation (3.17), each corresponding to a different set of boundary conditions. The theory we offer in this dissertation is among the most comprehensive of the possible applications in the sense that we embody in the scalar field the entire material content of the universe. As an alternative, equation (3.17) also could be used (with correspondingiy different boundary conditions) in a model where the scalar field was only onc of severa! components to the total energy.

In broad outline, the model presented her: resembles the model of Israclit and Rosen (1989) in that it colisists of prematter-, radiation:-, and matter-cominated epochs. In our notation below, the subscripts $p, r$, and $m$ refer to these epochs, respectively. As we have already noted, $\gamma_{m}=1$ and $\gamma_{r}=4 / 3$. We shall consider the physical consequences of several different values for $\gamma_{p}$.

The Lagrangian of the field theory for the entire history of the universe is determined once we find $V(\phi)$ for each epoch. The general solution or $V(\phi)$ with $\gamma$ equal to a constant has already been determined in equation (3.17). It remains for us to apply this result to our sperific model, and to determine the values of 
the constants $b, \alpha, \beta$, and $C$ for each epoch. The constants $b$ and $\alpha$ are obtained directly from their definitions in terms of $\gamma$ (see eq. [3.10b] and eq. [3.14b]). The constants $\beta$ and $C$ will be determined by the application of the boundary conditions.

The boundary conditions also allow determination of the initial and critical values of the scalar field. The initial value of $\phi$ is denoted as $\phi_{0}$, the quantity $\phi_{p r}$ is the critical value of $\phi$ at the prematter-to-radiation phase transition, and $\phi_{r m}$ is the critical value of $\phi$ at the radiation-to-matter transition. We reiterate a point mentioned in the Introduction concerning our use of the term "phase transition." In this work, a phase transition is a discontinuous change in the equation of state of the scalar field. In other models, the phase transition concept is used to describe a symmetry breaking of the vacuum state of a scalar field potential in accordance with the generalized theory of phase transitions due to Landau (see Linde 1979 for a comprehensive review). This latter interpretation of "phase transition" sas no relevance to the present work.

As a convenient choice for one of the boundary conditions in our theory, we set $\phi=0$ at the point of maximum expansion in the scale factor, at which point the density attains a minimum value. From equation $(2.12 b)$, we then see th $\phi=0$ corresponds to a minimum in the potential $\left(V_{m_{i n}}\right)$ whose value may be determined from the theory. Because of the perfect fluid assumption, the collapse 
of the universe is symmetric with its expansion. Therefore wo shall only consider the case of expansion.

In our model, $\gamma$ is discontinuous at the critical values of the scalar field. However, the energy density $(\rho)$ and the scale factor $(R)$ must be continuons across each transition if the total energy is to be a continuous function of $\phi$. Equation (2.12b) then yields discontinuities in $V(\phi)$ at $\phi_{r m}$ and $\phi_{p r}$. In particular, for two phases (which we denote in general as $I$ and $I I$ ), the potentials on either side of the transition are related according to

$$
V_{I}=\left[\frac{2-\gamma_{I}}{2-\gamma_{I I}}\right] V_{I I}
$$

In the equations below, we shall denote the values of the potential on the prematter and radiation sifles of $\phi_{p r}$ as $\left(V_{p r}\right)_{r}$ and $\left(V_{p r}\right)_{r}$, respectively. Similarly, the values of the potential on the radiation and matter sides of $\phi_{r m}$ are denoted as $\left(V_{r m}\right)_{r}$ and $\left(V_{r m}\right)_{m}$, respectively.

We must next consider the behavior of $d \ln V / d \phi$ at the critical values of $\phi$. Since $\gamma \rho=\dot{\phi}^{2} \neq 0$ (see eqns. [2.6] and [2.7]), equation (2.14) may be written as

$$
\rho^{\prime}=-3 H \dot{\phi}
$$

The continuity of $\rho$ and $R$ implies that the Hubble parameter is continuous across a phase transition as well (see eqn. $[2.10]$ ), and this allows us to write

$$
\left(\frac{d \ln \rho}{d \phi}\right)_{I}=\left[\frac{\gamma_{I}}{\gamma_{I I}}\right]^{1 / 2}\left(\frac{d \ln \rho}{d \phi}\right)_{I I}
$$


which, with equation ( $2.12 \mathrm{~b})$, becomes

$$
\left(\frac{d \ln V}{d \phi}\right)_{I}=\left[\frac{\gamma_{I}}{\gamma_{I I}}\right]^{1 / 2}\left(\frac{d \ln V}{d \phi}\right)_{I I}
$$

Equations (2.21), (3.16)-(3.18), (4.1), and (4.4) then provide the boundary conditions. At $\phi=0$ and $t=t_{m}$ :

$$
V_{\min }=C_{m}\left[1+\beta_{m}\right]^{1 / b_{m}}
$$

and

$$
\frac{\alpha_{m}}{b_{m}}\left[\frac{1-\beta_{m}}{1+\beta_{m}}\right]=0
$$

at $\phi=\phi_{r m}$ and $t=t_{r}$ :

$$
\left(V_{r m}\right)_{m}=C_{m}\left[e^{-\alpha_{m} \phi_{r m}}+\beta_{m} e^{\alpha_{m} \phi_{r m}}\right]^{1 / b_{m}}=\frac{3}{2} C_{r}\left[e^{-\alpha_{r} \phi_{r m}}+\beta_{r} e^{\alpha_{r} \phi_{r m}}\right]^{1 / b_{r}}
$$

and

$$
\frac{\alpha_{m}}{b_{m}}\left[\frac{1-\beta_{m} e^{2 \alpha_{m} \phi_{r m}}}{1+\beta_{m} e^{2 \alpha_{m} \phi_{r m}}}\right]=: \frac{\sqrt{3}}{2} \frac{\alpha \cdot}{b_{r}}\left[\frac{1-\beta_{r} e^{2 \alpha_{r} \phi_{r m}}}{1+\beta_{r} e^{2 \alpha_{r} \phi_{r m}}}\right]
$$

at $\phi=\phi_{p r}$ and $t=t_{p}$ :

$$
\left(V_{p r}\right)_{r}=C_{r}\left[e^{-\alpha_{r} \phi_{p r}}+\beta_{r} e^{\alpha_{r} \phi_{p r}}\right]^{1 / b_{r}}=\frac{1}{3}\left(\frac{2}{2-\gamma_{p}}\right) C_{p}\left[e^{-\alpha_{p} \phi_{p r}}+\beta_{p} e^{\alpha_{p} \phi_{p r}}\right]^{1 / b_{p}}
$$

and

$$
\frac{\alpha_{r}}{b_{r}}\left[\frac{1-\beta_{r} e^{2 \alpha_{r} \phi_{p r}}}{1+\beta_{r} e^{2 \alpha_{r} \phi_{p r}}}\right]=2\left(3 \gamma_{p}\right)^{-1 / 2} \frac{\alpha_{p}}{b_{p}}\left[\frac{1-\beta_{p} e^{2 \alpha_{p} \phi_{p r}}}{1+\beta_{p} e^{2 \alpha_{p} \phi_{p r}}}\right]
$$


at $\phi=\phi_{0}$ and $t=0$ :

$$
\frac{2-\gamma_{p}}{2}=C_{p}\left[e^{-\alpha_{p} \phi_{0}}+\beta_{p} e^{\alpha \phi_{p} \phi_{0}}\right]^{1 / b_{p}}
$$

and

$$
\frac{\alpha_{p}}{b_{p}}\left[\frac{1-\beta_{p} e^{2 \alpha_{p} \phi_{0}}}{i+\beta_{p} e^{2 \alpha_{p} \phi_{0}}}\right]=0 .
$$

The quantities $t_{p}$ and $t_{r}$ denote the times at which the two phase transitions occur, while the quantity $t_{m}$ denotes the time of maximum expansion in $R$. We have used $\gamma_{m}=1$ and $\gamma_{r}=4 / 3$, but we have not specified a (constant) value for $\gamma_{p}$. If $\gamma_{p}, V_{r m}$, and $V_{p r}$ are specified, equations (4.5) comprise a total of 10 equations in 10 unknowns $\left(\beta_{m}, \beta_{r}, \beta_{p}, C_{m}, C_{r}, C_{p}, \phi_{r m}, \phi_{p r}, \phi_{0}\right.$, and $\left.V_{m i n}\right)$.

The lifetime $(\Delta t)$ of each epoch may be determined once equations (4.5) are solved. With $\gamma$ equal to a constant, equations (3.17) and (3.20) yield

$$
\Delta t=\frac{1}{2 \alpha}\left[\frac{2-\gamma}{2 \gamma C}\right]^{1 / 2} \int_{x_{f}}^{x_{i}}\left[\frac{x^{1-4 b}}{(1+\beta x)^{2}}\right]^{1 / 4 b} d x
$$

where $x \equiv \exp [2 \alpha \phi]$, and the subscripts $i$ and $f$ refer to the inital and final values for the given epoch. Since $\phi$ evolves from a positive initial value $\left(\phi_{0}\right)$ and reaches zero at the point of maximum expansion in $R$, we have used the minus sign in equation (3.20). The times which are noted with regard to equations (4.5) are $t_{m} \equiv \Delta t_{m}+\Delta t_{r}+\Delta t_{p} ; t_{r} \equiv \Delta t_{r}+\Delta t_{p} ;$ and $t_{p} \equiv \Delta t_{p}$. In accordance with the general outline of the standard cosmological mordel, we would expect $\Delta t_{m}>\Delta t_{r}>\Delta t_{p}$ 
It is convenient to adopt the following notation:

$$
\begin{aligned}
x_{m r m} & \equiv \exp \left[2 \alpha_{m} \phi_{r m}\right], \\
x_{r r m} & \equiv \exp \left[2 \alpha_{r} \phi_{r m}\right], \\
x_{r p r} & \equiv \exp \left[2 \alpha_{r} \phi_{p r}\right], \\
x_{p p r} & \equiv \exp \left[2 \alpha_{p} \phi_{p r}\right], \\
x_{0} & \equiv \exp \left[2 \alpha_{p} \phi_{0}\right],
\end{aligned}
$$

and

$$
0<y \equiv \frac{x_{0}}{x_{p p r}}<1
$$

The upper limit in equation (4.8) arises because $0<\gamma_{p}<2 / 3$ implies $\alpha_{p}<0$, while $\phi_{0}>\phi_{p r}$ from the boundary conditions.

Using equations (3.10b) and (3.14b), we obtain

$$
\begin{aligned}
& b_{m}=\frac{1}{6} ; \quad \alpha_{m}=\frac{\sqrt{\kappa}}{2} ; \\
& b_{r}=\frac{1}{4} ; \quad \alpha_{r}=\frac{\sqrt{3 \kappa}}{2}
\end{aligned}
$$

and

$$
b_{p}=\frac{3 \gamma_{p}-2}{6 \gamma_{p}} ; \quad \alpha_{p}=3 b_{p} \sqrt{\kappa \gamma_{p}}
$$

The quantities $V_{r m}$ and $V_{p r}$ now may be specified on either side of the corresponding phase transition using equations $(2.12 \mathrm{~b}),(2.22),(4.1)$ and $(4.5)$. In 
particular,

$$
\left(V_{r m}\right)_{m}=C_{m} \frac{\left(1+\beta_{m}, x_{m r m}\right)^{6}}{x_{m r m}^{3}}=\frac{A \pi^{2}}{30}=\frac{3}{2}\left(V_{r m}\right)_{r}
$$

and

$$
\left(V_{p r}\right)_{r}=C_{r} \frac{\left(1+\beta_{r} x_{r p r}\right)^{4}}{x_{r p r}^{2}}=\frac{\pi^{2}}{45}=\frac{2}{3\left(2-\gamma_{p}\right)}\left(V_{p r}\right)_{p}
$$

After several calculations and substitutions, equations (4.5) yicld the following results:

$$
\begin{aligned}
& (1+y) y^{-1 / 2}=2\left[3\left(V_{p r}\right)_{r}\right]^{b_{p}} \\
& \frac{x_{m r m}}{\left(1+x_{m r m}\right)^{2}}=\frac{y}{(1+y)^{2}}\left[\frac{3\left(V_{p r}\right)_{r}}{2\left(V_{r m}\right)_{m}}\right]^{1 / 2} \\
& \phi_{r m}=\frac{1}{2 \alpha_{m}} \ln x_{m r m} \\
& \beta_{r}=x_{m r m}^{1-\sqrt{3}} \\
& C_{r}=\frac{2}{3}\left(V_{r m}\right)_{m} \frac{x_{m r m}^{2 \sqrt{3}}}{\left(1+x_{m r m}\right)^{4}} \\
& \beta_{m}=1 \\
& C_{m}=\left(V_{r m}\right)_{m} \frac{x_{m r m}^{3}}{\left(1+x_{m r m}\right)^{6}} \\
& x_{r p r}=\frac{1}{y \beta_{r}} \\
& \phi_{p r}=\frac{1}{2 \alpha_{r}} \ln x_{r p r} \\
& x_{0}=y x_{p p r} \\
& \phi_{0}=\frac{1}{2 \alpha_{p}} \ln x_{0}
\end{aligned}
$$




$$
\begin{gathered}
\beta_{p}=\frac{1}{x_{0}} \\
C_{p}=\left(\frac{2-\gamma_{p}}{2}\right) \frac{x_{0}^{1 / 2 b_{p}}}{2^{1 / b_{p}}}
\end{gathered}
$$

and

$$
V_{\min }=64 C_{m}
$$

Since $\left(V_{p r}\right)_{r}$ and $\left(V_{r m}\right)_{m}$ are specifed in equations (4.10), equations (4.11) may be solverl if $\gamma_{p}$ is specified. That is, $\gamma_{p}$ is the only remaining free parameter in the theory.

With regard to the lifetimes of the three epochs, equation (4.6) yields

$$
\Delta t_{m}=\left[\frac{3}{4 \pi}\right]^{1 / 2} V_{m i n}^{-1 / 2}\left[\frac{\left(x_{m r m}-1\right) x_{m r m}^{1 / 2}}{\left(x_{m r m}+1\right)^{2}}+\tan ^{-1}\left(x_{m r m}^{1 / 2}\right)-\frac{\pi}{4}\right]
$$

for the lifetime of the matter epoch, and

$$
\Delta t_{r}=\left[\frac{1}{32 \pi}\right]^{1 / 2} \frac{1}{\beta_{r} C_{r}^{1 / 2}}\left[\frac{1}{1+\beta_{r} x_{r r m}}-\frac{1}{1+\beta_{r} x_{r p r}}\right]
$$

for the life' ime of the radiation epoch.

In considering the lifetime of the prematter epoch, we first note that a combination of equations (4.10), (4.11a), and (4.11b) yields

$$
\frac{x_{m r m}}{\left(1+x_{m r m}\right)^{2}}=\frac{1}{4 A^{1 / 2}}\left[\frac{15}{\pi^{2}}\right]^{2 b_{p}} \text {. }
$$

Using $A=1.7539 \times 10^{-115}$ (see eqn. [2.22a]) and equation (4.9c) for $b_{p}$, it is possible to solve for $x_{m r m}$ if $\gamma_{p}$ is specified. As an example, if we were to let 
$\gamma_{p}=1 / 3$, we would find that $x_{m r m}$ is a complex number - a result that elearly is an unphysical solution for $x_{m r m}$ given its definition in equation (4.7a). Further investigation shows that a physically meaningful solution for $x_{m r m}$ is found only if $\gamma_{p}<<1$. It should be emphasized that this limitation on $\gamma_{p}$ is a consequence of the boundary conditions in equations (2.22) and (4.10) which, in turn, arose from our appeal to the standard cosmological model. Therefore, while any $\gamma_{p}<2 / 3$ may, in principle, provide inflation, only $0<\gamma_{p}<<1(\rho \approx-p$ in the carly universe) yields a universe whose later epochs resemble the standard cosmological model.

Consequently, when evaluating equation (4.6) for the lifetime of the prematter epoch, it suffices to consider only the case where $\gamma_{p}<<1$. We then ohtain

$$
\Delta t_{p} \approx-\frac{\kappa^{-1 / 2}}{2} \ln y
$$

for the lifetime of the prematter epoch.

We have now developed the theory in sufficient detail to be able to consirler specific cosmological results, and this is the subject of Chapter 5. 


\section{Chapter 5}

\section{Results and Discussion}

With $V_{r m}$ and $V_{p r}$ specified by equations (4.10), the only remaining free parameter in the entire theory is $\gamma_{p}$. For a given $\gamma_{p}$, the first task is to solve equations (4.11) for the other parameters in the theory, and the results corresponding to several different values of $\gamma_{p}$ are presented in Table 1 . The cosmological consequences then follow from equations $(2.12),(2.21),(2.23),(3.17)-(3.19),(4.12),(4.13)$, and (4.15), and these results are presented in Tables $2-8$.

In these tables, $\phi$ is given in units of the Planck mass $\left(m_{p l}\right)$, the scale factor $(R)$ is expresed in $c m$, the temperature $(T)$ is expressed in degrees Kelvin, and the density is given in $g m / \mathrm{cm}^{3}$. The quantities $C_{m}, C_{r}, C_{p}$, and $V_{\min }$ are given in units of the Planck density, and the present value of the Hubble parameter $\left(H_{0}\right)$ is given in $k m \sec ^{-1} M p c^{-1}$. The conversion factors between Planck units and cgs units are given in Appendix A.

Tables $2-7$ give results for $t=t_{p}, t=t_{r}$, and $t=t_{m}$ as well as for six values of $t$ in the range of the estimated present age of the universe $(15-20 \mathrm{Gyr})$. The results for $t_{p}, t_{r}$, and $t_{m}$ follow from the above-mentioned equations. The results for the present age of the universe are found by first solving for $x_{f}$ in equation 
(4.6) with $\Delta t$ set equal to the present age. We also set $x_{i}=x_{m r m}$, and the other quantities in equation (4.6) assume their respective values for the matter epoch. Once we solve for $x_{f}$ - which here corresponds to the value of $x$ at the present age - the present value of $\phi$ then follows directly, and the above-mentioned cequatious are then used to solve for the other cosmological quantities.

The value for $H_{0}$ is obtained from equation (2.10) using the present value of the density as given in Tables $2-7$, and using $H_{0}=100 \mathrm{~h} \mathrm{~km} \mathrm{sec}^{-1} \mathrm{M} \mathrm{pr}^{-1}=$ $1.7470 h \times 10^{-61} t_{p l}^{-1}$. The results for $H_{0}$ are given in Table 8 . The lower and upper limits for $H_{0}$ correspond to the upper and lower limits of the present age of the universe (that is, 20 and 15 Gyr), respectively.

Once the potential is determined for a given $\gamma_{p}$, the initial temperature is found frem equation (3.19) using the fact that $T=T_{p l}$ at $t=t_{p}$, where $T_{p l}$ is given by equation (2.23). The initial temperatures are given in Table 8 for cach value of $\gamma_{p}$.

The fact that the initial equation of state affects the overall dynamical behavior of the later universe may at first seem puzaling in light of the familiar result that inflation "erases" any "memory" of other initial conditions, such as those initial conditions pertaining to the perturbation spectrum (the so-called cosmic "no-hair" theorem; see the discussion in Linde 1990). Inflation can indeed take a 
broad range of initial conditions for the perturbation spectrum and yield a seemingly smooth, homogeneous, and isotropic universe. However, the dynamics of the scale factor and, consequently, the overall density and the Hubble parameter, ase driven by the equation of state, and the initial value of $\gamma$ has lasting effects that are unique to a closed cosmological model.

If a closed universe began in a radiation-dominated state at the Planck density, it would quickly collapse. In the model presented here, however, the universe begins with $0<\gamma_{p}<<1$ and inflates by an amount which is regulated by the values of $\gamma_{p}$ and $T_{p l}$. Since $T_{p l}$ is fixed in terms of the fundamental constants, the value of $\gamma_{p}$ determines the subsequent behavior of the universe.

Qualitatively, the early universe in our theory must inflate $(\ddot{R}>0)$ from its initial size (on the order of a Planck length) to a large enough size ( $R$ is an absolute measure in a $k=+1$ model) in order that once the phase transition to radiation occurs (and $\ddot{R}$ becomes negative), both $R$ and $\dot{R}$ will be large enough $\left(H \equiv \dot{R} / R \approx(8 \pi / 3)^{1 / 2}\right)$ to insure that the universe wiil not quickly contract, but will instead continue to expand for at least $15-20 \mathrm{Gyr}$. A contraction phase is a characteristic of a closed cosmological model that is not shared by open or flat models. That is, for the closed, non-singular model that we describe in our theory, the extent of inflation determines how long the universe will continue to expand after inflation ends. 
However, the extent of inflation depends on both $T_{p l}$ (expressed in terms of the fundamental constants) and $\gamma_{p}$ (a free parameter) in the following way: 1) $\gamma_{p}$ affects $b_{p}$ (eq. $\left.\left.[4.9 \mathrm{c}]\right) ; 2\right) b_{p}$ affects $y$ (eq. [4.11a]) for a specified $\left(V_{p r}\right)_{r}$ (eq. $[4.10 \mathrm{~b}])$, and $\left(V_{p r}\right)_{r}$ is in turn determined by $T_{p l}($ ex. $[222 b]$ and the other definitions); 3) $y$ affects the duration of inflation $\Delta t_{p}$ (eq. [4.15]).

The curvature term in equation (2.10) will begin to dominate as the universe approaches its maximur size, just as it dominates when the universe is at its minimum size of the order of a Planck length. Only if there is sufficient inflation in the early universe will the later universe continue to expand for $15-20 \mathrm{~Gy}$ r and still appear flat at that time. The analysis of our theory shows that sufficiont inflation arises only if $\gamma_{p}$ is sufficiently small and, in particular, we find that there exists a range of values for $\gamma_{p}$ which yield a universe witi $i_{2}$ approximately the same values for $\rho$ and $H$ at $15-20 \mathrm{Gyr}$. This is illustrated in Tables 2-7, where, in particular, Tables 2 and 3 show this range for $\gamma_{p}$ to be $0<\gamma_{p} \leq \epsilon$, where $\epsilon \approx 1.9 \times 10^{-3}$.

The results presented in Tables 2 and 3 show that the present density should be in the range $2.00-3.55 \times 10^{-30} \mathrm{gm} / \mathrm{cm}^{3}$. In addition, decreasing the value of $\gamma_{p}$ lowers the initial temperature, increases the extent of inflation, and increases the age at which maximum expansion occurs. For example, if $\gamma_{p}=1.90 \times 10^{-3}$, the initial temperature is $T_{i}=4.50 \times 10^{-64}{ }^{\circ} \mathrm{K}$, the extent of inflation is a factor of 
approximately $10^{32}$, and the age of maximum expansion is $t_{\max }=5.97 \times 10^{15} \mathrm{yr}$. If, however, $\gamma_{\boldsymbol{r}}=1.85 \times 10^{-3}$, then $T_{i}=1.17 \times 10^{-66}{ }^{\circ} \mathrm{K}$, the extent of inflation is approximately $10^{33}$, and $t_{\max }=2.30 \times 10^{18} \mathrm{yr}$. The value of the scale factor at $15-20 \mathrm{Gyr}$ increases as the choice for $\gamma_{p}$ decreases. This is to be expected since a smaller $\gamma_{p}$ yields greater inflation in $R$ in the early universe.

We can set an upper limit on $\gamma_{p}$ by considering the age of maximum expansion. We find that $\gamma_{p}=2.02 \times 10^{-3}$ yields $T_{i}=2.17 \times 10^{-58}{ }^{\circ} \mathrm{K}$, and the extent of inflation is approximately $10^{30}$. However this modei reaches its maximum size after only 12.4 Gyr. Still larger values of $\gamma_{p}$ provide less inflation in the early universe and still lower ages at maximum expansion, thereby making such models unacceptable. For the acceptable models, the duration of the inflationary epoch is on the order of $10^{-42} \mathrm{sec}$, and the duration of the radiation era is approximately $8.7 \times 10^{5}$ yrs.

Tables 2 - 7 show that $R$ inflates during the prematter epoch by a factor of $10^{29}-10^{30}$. This is approximately the same amount of inflation that is found in most other theories. The constraints on the extent of inflation in other models arise indirectly from assumptions regarding fluctuations in the early universe and the evolution of those fluctuations into inhomogeneities of the matter and radiation distribution at the current epoch. In our theory, the extent of inflation is determined directly by the values of $\gamma_{p}$ and $T_{p l}$. It remains to be seen whether 
the consideration of fluctuations will impose ad.iitional constraints on the initial conditions.

Table 8 shows that the present value for the Hubble parameter $\left(H_{0}\right)$ should be in the range $33-44 \mathrm{~km} \mathrm{sec}^{-1} \mathrm{Mpc}^{-1}$. This range of values does have some overlap at the low end of the range of the most frequently cited values $(40-$ $100 \mathrm{~km} \mathrm{sec}^{-1} \mathrm{Mpc}^{-1}$ ), and it is instructive to review the observational techniques by which $H_{0}$ is determined.

From a practical point of view, the observational determination of the Hubble parameter reduces, in effect, to a determination of the cosmological distance scale, with a longer distances ale yielding a lower value for $H_{0}$. Cosmologiral distance indicators may be divided into two general classes ("primary" and "secondary") which are distinguished by the degree to which the astrophysical propertics of various objects are well-defined. Objects whose properties are well-understood are often referred to as "standard candles" when employed for the purposes of determining cosmological distances.

All methods aim, either directly or indirectly, at finding $\iota_{1} \mathrm{le}$ intrinsic: magnitude of the object under consideration from which a distance modulus may then be calculated. Primary indicators rely either on direct geometrical techniques (geometric parallax) or such well-defined (i)jects as RR Lyrac stars (main-sequence? 
fit" ing terhnigues) Cepheid variables (the period-luminosity relation), and normal novae (using the relation between the intrinsic magnitude and the rate of decline in the apparent luminosity of novae).

The distance to which primary indicators may probe is generally limited to a few megaparsecs; this should be compared to a horizon distince of approximately $1000 \mathrm{Mpc}$. The relative motions of the galaxies (approximately 10 in number) that lie within 3-4 megaparsecs are dominated by local peculiar motions and are not representive of the lange-scale expansion of the universe. In order to discern the underlying Hubble expansion, it is generally accepted that distances exceeding $10 M p c$ must be probed, and it is for this reason that distance determinations to the Virgo cluster (at approximately $15-22 \mathrm{Mpc}$ ) become so important. One of the most signifcant developments during the past several years (with regard to the determination of the Hubble parameter) has been the extension of the nova technique to the Virgo cluster by Pritcher and van den Bergh (1987).

Probing to cistances larger than $10 M p c$ requires the use of secondary indicators that rely upon less certain assumptions regarding the properties of the object observed. For instance, the Tully-Fisher relation for spiral galaxies and the Faber-Jackson relation for elliptical galaxies have been applied extensively to the Virgo cluster. The Tully-Fisher relation determines the velocity dispersion in the galaxy by measurement of the width of the $21 \mathrm{~cm}$ spectral line of neutral 
hydrogen. It is based upon a reasonably well-established empirical correlation between the internal motions of gas-rich spiral galaxies and intrinsic galactic luminosity; the physical rationale being that each characteristic is related to the mass of the galaxy, with more massive galaxies being zerore luminous and rotating more quickly. The Faber-Jackson relation has a similar physical rationale for the more gas-poor elliptical galaxies. Here the relationship is between total galactic luminosity and velocity dispersion near the center of the galaxy, where it is implied that all elliptical galaxies have approximately the same mass to-light ratio.

Other techniques exist as well. Many employ morphological properties of galaxies or the brightest galaxies in clusters in correlation with galactic or cluster mass. Supernovae and planetary nebulae have similarly boen employed in an effort to extend the cosmological distance ladder to ever-farther distances. An introductory account of primary and secondary techniques may be found in Rowan-Robinson (1985). A more complete account of Virgo cluster techniques is Sandage and Tammann (1990).

Until re ntly, most methods had seemed to converge on a distance to the Virgo cluster of approximately $15 M p c$. Given the observed recession velocity of approximately $1300 \mathrm{~km} / \mathrm{sec}^{-1}$, this distance corresponds to a Hubble constant of approximately $87 \mathrm{~km} \mathrm{sec}^{-1} \mathrm{Mpc}^{-1}$. However Sandage and Tammann (1990) employed six independent techniques to derive a distance of approximatcly $22 \mathrm{Mpc}$. 
By further removing local velocity anomalies from the velocity of the Virgo cluster, they found a recession velocity of approximately $1140 \mathrm{~km} / \mathrm{sec}^{-1}$. These results then yield $H_{0} \approx 52 \mathrm{~km} \cdot \mathrm{sec}^{-1} \mathrm{Mpc}^{-1}$.

Still more recently Sandage (1993) compared eleven methods and found that nine favor the longer distance scale, including one morphological technique that surveys 86 field galaxies and yields $H_{0} \approx 43 \pm 11 \mathrm{~km} \mathrm{sec}^{-1} \mathrm{Mpc}^{-1}$. In addition, recent Cepheid variable studies of IC 4182 with the Hubble Space Telescope also argue for the longer distance scale, with $H_{0} \approx 51 \pm 10$ (Sandage, et al. 1992).

Thus, there still exists substantial disagreement over the value of $H_{0}$ despite some claims to the contrary. Lower values of $H_{0}$ imply an older expansion age for the universe $\left(t_{\varepsilon x p} \sim 1 / H_{0}\right)$. A value of $H_{0}=87 \mathrm{~km} \mathrm{sec}^{-1} \mathrm{Mpc}^{-1}$ corresponds to an expansion age of approximately $7.5 \mathrm{Gyr}$ under the assumption of a $k=0$ FRW model with no cosmological constant. However the oldest star clusters in our galaxy have ail age of $13-18 \mathrm{Gyr}$, and must therefore set a lower limit on the age of the universe. Until the past year, the shorter distance scale was gaining favor. However the most recent and most extensive surveys of distance-measuring techniques have favored the longer distance scale, thereby giving an estimate for $H_{0}$ that is at the lower end of the generally-accepted range. The value of $H_{0}$ predicted by our theory $\left(33-44 \mathrm{~km} \mathrm{sec}^{-1} \mathrm{Mpc}^{-1}\right)$ is therefore reasonable. 
For the remainder of this Chapter, we discuss several other issues mentioned over the course of this dissertation: 1) the thermodynamic limitations on a more complete theory of matter for the early universe; 2) the phase transitions at critical field values; and 3) singularity-avoidance upon the recollapse of the universe.

1) Our theory describes a universe that is initially very cold .... a result also obtained by Israelit and Rosen (1989). The mechanism by which the carly universe is heated is shown to follow from the requirements that a regative pressure exists in the early universe and that the first and second laws of thermodynamies be satisfied (see eq. [2.20]). The universe continues to inflate and heat until the maximum temperature is reached, at which point the scalar field undergoes a phase transition to a radiative equation of state.

It is significant that no excessive fine-tuning is required to stop inflation and reheat the early miverse. The issue of how inflation ends and a radiationdominated universe reheats has been a central question in contemporary theoretical cosmology. In our theory, inflation and heating are accomplished by appealing to very basic considerations, and inflation ends when a maximum physical temperature is attained. Further, this maximum temperatue follows from a natural scaling in terms of the fundamental Planck units. Unlike other theories, vihere the need for a reheating mechanism arises because of inflation, our theory shows that inflation and heating are compatible characteristics of the early universe. 
In order for this feature of our theory to be relevant to the observed universe, the thermal tehavior of the scalar field in our theory must be related to the thermal behavior of a radiative component in the actual universe. Although the numerical details would be different, a more robust cosmological fluid may be superimposed upon the scalar field in our theory while retaining the general features described above. In this regard, it would be necessary to consider at least the fact that our model is adiabatic, thereby requiring $g_{* s} T^{3} R^{3} \propto S$ to be constant, where $S$ is the total entropy, and $g_{* s}$ is a temperature-weighted sum of the number of relativistic degrees of freedom in the mixture of fermions and bosons (including photons) which would comprise the cosmological fluid. For example, when the standard cosmological model is combined with the standard model of particle physics, $g_{* s}$ decreases from approximat $1 \mathrm{ly} 106.75$ in the early universe to approximately 3.91 at the current epoch (Kolb and Turner 1990).

As the temperature and scale factor increase during the inflation epoch, $g_{* s}$ would have to decrease in order for the total entropy to remain constant. Because of the very large increases in both the scale factor and the temperature during the inflation era, $g_{* s}$ initially would have to be an extremely large number in order for it to still be as large as $\sim 10^{2}$ at the beginning of the radiation era. After inflation, the photons would remain in equilibrium with the scalar field up to the time of decoupling - the temperature of each decreasing as $1 / R$ during the radiation 
era. After decoupling, the scalar field would remain isothermal (see the disceussion below) while the temperature of the radiation would continue to decrease as $1 / R$.

The ciecrease in $g_{* s}$ during the inflationary epoch is interpreted here as a thermodynamic constraint that must be met by a more complete theory of matter for the early universe if that theory is to accoilii for the present photon temperature and still remain consistent with the general results of our theory. This behavior for $g_{* s}$ is unlike that for matter and radiation under ordinary conditions, but this early phase - characterized by a negative pressure -... may well admit this behavior. This early phase also possesses a negative specific heat; that is, its temperature increases while its density decreases. Negative specific heats are common in more familiar astrophysiral settings (Lynden-Bell and Lynden-Bell 1977; Thirring 1970), although we do not suggest that the same mechauism th it accounts for negative specific heats in, say, an isolated star, also accounts for this feature in our cosmological theory.

One implication of a very large value for $g_{* s}$ in the early universe is with regard to the number of particle species present. The definition of $g_{* s}$ (Koll) and Turner 1990) is such that we are required to postulate a very large number of elementary particles in the early universe. This may be a cause of theoretical discomfort in the context of the standard model for particle physics, but it is not illogical to assume that ever-higher energies will continue to reveal still more 
structure in much the same way that we have come to observe structure in particles once thought to be elementary.

The constraint on $g_{* s}$ arose in part from the assumption that the total entropy of the universe is conserved. It is interesting to ask how the situation may change if an entropy-generation mechanism were introduced into the theory. As we discussed in Chapter 2, one means of doing this is through the introduction of a bulk viscosity term in the energy-momentum tensor. It is certainly possible to construct viscous properties of the cosmological fluid in our model in such a way as to not only generate entropy for each cycle of universal expansion and contraction, but in yield a total entropy that is asymptotically finite as the universe evolves through an infinite number of cycles. These conditions would necessarily serve as a constraint on the properties of the scalar field in our theory.

2) With regard to the phase transitions, our theory provides for the determination of the critical values of the scalar field at which these transitions occur. These critical field values, which our results show to be in the approximate range $2-34 m_{p l}$ (see Tables $2-7$ ), may be interpreted as mass scales in our theory, although the physical significance of these mass scales remains to be determined. Nonetheless, some speculations can be made along these lines.

The phase transitions denote the boundaries between cosmological epochs, 
which themselves may be characterized in several different, but equivalent, ways. We can think of the epochs in terms of the phase of the scalar field, or in terms of the time evolution of the scale factor, or in terms of the time evolution of the temperature.

Ultimately, the physical significance of the critical values of the scalar field will depend upon a clearer resolution of the physical interpretation of the field itself. From the outset, our theory is premised upon continuum physics in that we treat the scalar field as a classical continuum. We even suggested in Chapter 2 that entropy production might be accomodated through a bulk viscosity term in the energy momentum tensor, although we have considered only the perfect fluid assumption in this dissertation.

In the "thermudynamic constraint" analysis given above, we envisioned the scalar field as a "substrate" upon which recognizable forms of matter may be superimposed. We derived a particular behavior (the behavior of $g_{* s}$ ) that matter in the early universe must obey if the theory we present is to account for the universe we actually observe. This interpretation of the scalar ficld as a substrate suggests that we interpret the scalar field as a dark matter component.

The dark mi tter that is postulated to exist in the actual universe must interact very weakly with ordinary matter and radiation under the conditions of the 
present epoch, for otherwise it would have been detected long ago. However, it would not be expected that this interaction would necessarily have always been so weak. We see these very attributes in the scalar field of our theory most apparently when we consider the temperature evolution of the field over the course of the three epochs. Equation (2.20) shows that the temperature increases with the expansion of the scale factor if $\gamma<1$ and constant. This condition is satisfied during the prematter epoch in our model. After the transition to the radiative phase, the temperature of the scalar field decreases as $1 / R$. To this point of the evolution, the scalar field has remained in equilibrium with whatever other material components might be superimposed upon the scalar field. However, during the subsequent matter epoch, the temperature of the scalar field remains constant -- a consequence of our assumption that $\gamma=0$ and is constant during the matter era (see eqn. [2.20]). This behavior is very unlike the behavior of the kinetic temperature of ordinary material particles during the matter era of the standard model (where $T_{k i n} \sim 1 / R^{2}$ ).

Consequently, during the matter era, the scalar field in our theory is not physically quivalent to a mixture of nonrelativistic particles, and one can argue that the scalar field in our theory - much like the elusive dark matter which all other inflation theories presume to exist in the actual universe - is very much unlike ordinary matter as we know it. 
Therefore, whatever may prove to be the best physical interpretation of the critical values of the scalar field in our theory, perhaps they can be related to certain properties of the dark matter.

3) In our theory, the radiation and matter epochs follow the general outline of the standard model, with the material content described by the scalar feld. Eventually, the universe reaches a maximum size, contracts to its initial sizc, and continues to oscillate. Throughout the development of our theory, we have adopted the usual idealization that the universe is homogeneous and isotropic. This assumption is important with regard to singularity-avoidance upon the contraction of the universe. If spatial gradients of the scalar field are included in the Lagrangian, these gradients would grow and could dominate dhuing a "defla-tionary" prematter stage (Altshuler 1990). The thermodynamic offect would be an equation of state which would force a closed model into a singularity during a recontraction phase (Kolb and Turner 1990). A proper consideration of spatial gradients of $\phi$ would require that we depart from strict adherence to the FRW metric, and therefore singularity-avoidance should be considered in context with the treatment of anisotropies in our classical field theory. In a model much different from ours, a means for establishing a stable deflationary epoch has beon suggested by Altshuler (1990).

This discussion of the bchavior of spatial gradients in the scalar field during 
deflation raises a more general issue regarding the asymptotic stability of spatially homogeneous cosmological models. Any homogeneous space admits a group of transformations, and the group structure constants may be decomposed in some basis. Such a decomposition forms the foundation of the Bianchi classification scheme for homogeneous cosmological models (see Landau and Lifshitz 1975).

The asymptotic $(t \rightarrow \infty)$ stability of Bianchi-type universes has been studied extensively. The general approach of the stability analysis is to first evaluate the eigenvalues of a system of linearized equations that represent perturbations to the unperturbed solutions. For each Bianchi type, the stability analysis must be specialized to the properties of the energy-momentum tensor (such as whether the unperturbed solution is a vacuum or perfect-fluid solution), the equation of state, and the perturbation characteristics. In most instances this linearization analysis is insufficient, and higher-order perturbations must be taken into account in order to render a more reliable conclusion regarding the asymptotic stability of the space. Stability analysis in general relativity is a nontrivial issue for even the simplest spaces. Fortunately, for our purp - as here, a large body of work exists in the literature to which we may refer. Among the earliest works are Collins (1971) and Collins and Hawking (1973). An extensive review of perturbation techniques and stability properties of different Bianchi-type universes may be found in Barrow and Sonoda (1986). 
As isotropic spacetimes, the FRW models are special cases of various Bianchitype universes. In particular, the $k=0 \mathrm{FRW}$ model is a special case of Binnchitype $I$ and type $V I I_{0}$ universes; the $k=-1$ FRW model is a special case of type $V$ and type $V I I_{h}$; and the $k=+1$ FRW model is a special case of a Bianchi-type $I X$ universe (Barrow and Sonoda 1986; Collins and Hawking 1973). The $k=0$ FRW model is an example of a relatively simple homogeneous space wherein the results of a stability analysis depend on the assumed details. For instance, Barrow and Sonoda (1986) have shown this model to be asymptotically stable for a co-moving perfect fluid with $\gamma \leq 5 / 4$; Barrow and Tipler (1978) have shown this same model to be unstable to non-comoving velocity perturbations if $\gamma \geq 4 / 3$; Collins and Hawking (1973) have shown the model to be asymptotically stable against noncomoving velocity perturbations if $\gamma=1$. A similar variety of stability results exists for the open $k=-1$ FRW models as well.

Our particular interest is with regard to the closed FRW models, and here too the analysis for anisotropic perturbations has been extensive. These closed anisctropic models are the so-called "mixmaster models" (Misner, et al. 1973) which received a great deal of attention during the 1970's and carly 1980's. In particular, Barrow (1982) has shown that if a Bianchi-type; $I X$ universe begins near an FRW state (but with a small anisotropic perturbation) and evolves toward a singularity, then that singularity will exhilit chaotic, mixmaster behavior. 
Our theory provides for singularity avoidance so long as the universe remains isotropic. However we have noted that if spatial gradients of the scalar field are included in the Lagrangian of our theory then these gradients grow and could dominate during deflation and force our closed model into a singularity. We also see that anisotropies would grow during the radiation and matter epochs as well, since these are the epochs in our closed model that are, in effect, the subject of the Barrow analysis. Hence, anisotropies would be expected to grow during the evolution of the universe described by our theory.

Therefore the next step in the development of our theory should be an extensive investigation of the extent to which anisotropies can grow within the context of the theory we have described while still preserving a singularity-free universe. After all, the matter in the universe must ultimately form structures on scales at least as large as superclusters. The challenge of a further study will be to accomodate the observed anisotropies within the unified framework offered for the first time by our theory.

In a much different vein, it may be possible to avoid a singularity by applying the idea of Markov and Mukhanov (1984), whereby the gravitational interaction is asymptotically-free at very high energies. In addition to its original cosmological applications, this idea has been applied to the issue of singularity avoidance in black holes (Frolov, et al. 1990). This approach would, however, take us outside 
the realm of Einstein's theory, and other features of our morlel would be affected were we to adopt a variable $G$ in the carly universe. Interestingly, variable-G theories for the early universe have gained renewed interest in the form of "extended" and "hyperextended" inflation models (La and Steinhardt 1989; Stcinhardt and Accetta 1990).

We note that equation (3.17) for $V(\phi)$ is suggestive of, but not equivalent to, the potentials associated with power-law inflation theories (Olive 1990). It also should be noted that our derivation of equation (3.9) may be altered slightly to yield an equation for $\gamma(\phi)$ if $V(\phi)$ is known. This should be of interest in those circumstances where a postulated form for $V(\phi)$ is being tested for its cosmological consequences.

The issues discussed in this chapter compel us to inquire as to the nature of the observations that might be made to confirm our theory. Any measured value of the Hubble parameter or the critical density (see Appendix B) may be accomodated by one inodel or another from within the stindard cosmological framework wherein the universe evolves through various epochs. Further, our estimates for $H_{0}$ are consistent with the predictions of any morlels wherein the universe has closely resembled a $k=0$, matter-dominated FRW model for most of its lifetime including the present epoch. The principal feature of our theory that distinguishes it from other models is its unified description of all cosmologcial 
cpochs by means of a single scalar field to represent the encrgy content of the universe. Other features, such as the absence of a singularity, follow from the physical propertios asc ribed to the field. Therefore, those observations that are most relevant to our theory are those that reveal the physical properties of the inaterial content of the universe.

Perhaps the most intriguing aspect of this work is the strong suggestion that the scalar field in our theory is physically related to the dark matter content of the universe. Consequently, the most relevant astronomical observations are those pertaining to the cosmological distribution of dark matter. It is reasonable to expect that the distribution of dark matter, and the environments in which it is fouml, will ultimately reveal much information about the nature of this material substance that is generally thought to comprise 90 percent of the energy content of the universe. Further, the most unfamiliar property of the scalar field in our theory is that it represents a cosmological fluid which must attain an equation of state $p \approx-\rho$ at an early epoch. Therefore, an important observation or theoretical development with regard to our theory would be the confirmation that the dark matter would attain this equation of state under the extreme conditions of the carly universe. 


\section{Chapter 6}

\section{Summary}

The theory that we have presented Irovides a greater sonse of rontinuity between the early and late epochs of the universe than what has becn obtained thusfar from other contemporary cosmological theories. This is becausc our field theory for the material content of the universe is not restricted in its application to an early epoch, but is applied to all cosmological eras. Conserquently, a Ligrangian function is found that describes the evolution of the universe throughout its entire history.

Our theory invokes the simplifying assumption that the matcrial content of the universe begins with a "vacuum-like" equation of state. Wo then olstain a cosmological theory without a singularity in which an inflaticnary epoch arise's as a consequence of boundary conditions which are expressed in terms of the fundamental constants of nature (the Planck quantities). This is unlike other theories which depend on the existence of a pre-inflationary, radiation dominated universe, and in which tinc boundary conditions for this pre-inflationary epoch include a sing 'larity.

In our theory, heating is compatible with inflation, and the theory identifies 
a physical state - - the attainment of a maximum possible physical temperature that marks the end of the inflationary era. This is in marked contrast to other models where the inclusion of an inflationary epoch forces the subsequent imposition of a finely tuned mechanism in order to make the transition from the inflationary era to the standard model. We further derive a chermodynamic constraint that must be met by a more complete theory of matter for the early universe if that theory is to account for the present photon temperature and still remain consistent with our general results.

We demonstrate that inflation is necessary if the later epochs are to resemble the standurd model, and that there exists a range of initial equations of state near $p \approx-\rho$ which yield the same ubservable features for the present epoch. This absence of excessive fine-tuning is a desirable feature in any cosmological theory. We have derived the differential equation (whose form is that of Riccati's equation) which connects an arbitrary equation of state and the scalar field potential. This equation may be useful beyond its specific application in our theor: 


\section{Bibliography}

Altshuler, B. L. 1990, Class. Quantum Grav., 7, 189.

Barrow, J. D. 1982, Phys. Rep., 85, 1.

Barrow, J. D. and F. J. Tipler 1978, Nature, 276, 473.

Barrow, J. D. and D. H. Sonoda 1986, Phys. Rep., 139, 1

Bekenstein, J. D. 1992, in The Astronomy and Astrophysics Encyclopedia, ed. S. P. Maran (New York: Van Nostrand Reinhold), p. 296.

Börner, G. 1988, The Early Universe - Facts and Fiction (Berlin: SpringerVerlag).

Brandenberger, R. H. 1985, Rev. Mod. Phys., 57, 1.

Collins, C. B. 1971, Comm. Math. Phys., 23, 137.

Collins, C. B. and S. W. Hawking 1973, Ap. J., 180, 317.

Coilins, P. D. B., A. D. Martin, and E. J. Squires 1989, Particle Physics and Cosmology (New York: John Wiley and Sons).

Cooperstock, F. I. and N. Rosen 1989, Int. J. Theor. Phys., 28, 423.

Davis, H. T. 1962, Introduction to Nonlinear Differential and Integral Equations (New York: Dover).

Ellis, G. F. R. and M. S. Madsen 1991, Class. Quantum Grav., 8, 667.

Frolov, V. P., M. A. Markov, and V. F. Mukhanov 1990, Phys. Rev. D., 41, 383.

Gliner, E. B. 1966, Sov. Phys. JETP, 22, 378.

Guth, A. H. and M. Sher 1983, Nature, 302, 505.

Israelit, M. and N Rosen 1989, Ap. J., 342, 627. 
Kolb, E. and M. Turner 1990, The Early Universe (New York: Addison-Wesley). La, D. and P. J. Steinhardt 1989, Phys. Rev. Lett., 62, 376.

Landau, L. D. and E. M. Lifshitz 1975, The Classical Theory of Fields (4th Edition, New York: Pergamon Press).

Landau, L. D. and E. M. Lifshitz 1980, Statistical Physics - Part I (3rd Edition, New York: Pergamon Press).

Landan, L. D. and E. M. Lifshitz 1987, Fluid Mechanics (2nd Edition, New York: Pergamon Press).

Linde, A. D. 1979, Rep. Prog. Phys. 42, 389.

Liride, A. 1990, Particle Physics and Inflationary Cosmology (New York: Harwood).

Lynden-Bell D. and R. M. Lynden-Bell 1977, Mon. Not. R. Astr. Soc., 181, 405.

Markov, M. A. 1982, JETP Lett., 36, 265.

Markov, M. A. and V. F. Mukhanov 1984, JETP Lett., 40, 1043.

Misner, C. W., K. S. Thorne, and J. A. Wheeler 1973, Gravitation, (New York: W. H. Freeman asd Company).

Olive, K. A. 1990, Phys. Rep., 190, 308.

Pickering, A. 1984, Constructing Quarks - A Sociological History of Particle Physics (Chicago: University of Clicago Press).

Pritchet, C. J. and S. van den Bergh 1987, Ap. J., 318, 507.

Rowan-Robincon, M. 1985, The Cosmological Distance Ladder (Nerv York: W. H. Freeman and Company).

Sandage, A. and G. A. Tammann 1990, Ap. J., 365, 1.

Sandage, A., A. Saha, G. A. Tammann, N. Panagia, and D. Macchetto 1992, Ap. J. Lett., 401, LT.

Sandage, A. 1993, Ap. J., 402, 3. 
Smoot, G. F., C. L. Bennett, A. Kogut, E. L. Wright, J. Aymon, N. W. Boggess, E. S. Cheng, G. De Amici, S. Gulkis, M. G. Hauser, G. Hinshaw, P. D. Jackson, M. Janssen, E. Kaita, T. Kelsall, P. Keegstra, C. Lineweaver, K. Loewenstein, P. Lubin, J. Mather, S. S. Meyer, S. H. Moseley, T. Murdock, L. Rokke, R. F. Silverberg, L. Tenorio, R. Weiss, and D. T. Wilkinson 1992, Ap. J. Lett., 396, L1.

Starkovich, S. P. and F. I. Cooperstock 1992, Ap. J., 398, 1.

Steinhardt, P. J. and F. S. Accetta 1990, Phys. Rev. Lett., 64, 2740.

Thirring, W. 1970, Z. Physik, 235, 339.

Weinberg, S. 1972, Gravitation and Cosmology (New York: Jolnn Wiley and Sons). 


\section{Appendix A}

\section{Natural Units}

We adopt the natural system of units in which

$$
h=c=k_{B}=1,
$$

where $k_{B}$ is Boltzmann's constant. In these units, all of the fundamental physical quantities are expressed in terms of energy:

$$
[\text { energy }]=[\text { mass }]=[\text { temperature }]=[\text { length }]^{-1}=[\text { time }]^{-1}
$$

Within this system of 'mits, we still have the freedom to choose a mass scale, and in this dissertation we choose the Planck scale by setting the Planck mass equal to unity. Consequently, all of physical quantities calculated in our theory are expressed in terms of the corresponding Planck units. For example, in a closed cosmological model where the scale factor is a radius of the universe, that radius is measured in Planck lengths in our theory.

As another c:ample, a scalar field in the I agrangian formalism has physical dimensions of $(M L)^{\frac{1}{2}} / T$. In natural units, this reduces to energy or mass units, ard by further scaling our equations in Planck units, the value of $\phi$ in our equations is in units of the Planck mass. 
In a similar nanner, it is straightforward to show that the density, pressure, and scalar field potential that appear in our theory are all expressed in uuit. of the Planck density. In Chapter 2 we encounter expressions that involve cntropy density $(s)$ and a bulk viscosity coefficient $(\zeta)$, and even though we do not consider entropy production in our (adiabatic) theory, we note for completeness that the scaling of our equations implies that both $s$ and $\zeta$ would be expressied in units of $m_{p l}^{3}$.

In order to assist in the conversion of the results obtained in our theory to more familiar units, we provide in this Appendix those conversion factors, fundamental constants, and cosmological parameters that occur most frequently (adapted from Kolb and Turner 1990). 


\section{Conversion Factors:}

Energy: $1 \mathrm{GeV}=1.6022 \times 10^{-3} \mathrm{ergs}$

Temperature: $1 \mathrm{GeV}=1.1605 \times 10^{13} \mathrm{~K}$

Mass: $1 \mathrm{GeV}=1.7827 \times 10^{-24} \mathrm{~g}$

Length: $1 \mathrm{GeV}^{-1}=1.9733 \times 10^{-14} \mathrm{~cm}$

Time: $1 \mathrm{GeV}^{-1}=6.5822 \times 10^{-25} \mathrm{sec}$

Number Density: $1 \mathrm{GeV}^{3}=1.3014 \times 10^{41} \mathrm{~cm}^{-3}$

Mass Density: $1 \mathrm{GeV}^{4}=2.3201 \times 10^{17} \mathrm{~g} \mathrm{~cm}^{-3}$

Megaparsec: i $\mathrm{Mpc}=10^{6} \mathrm{pc}=3.0856 \times 10^{24} \mathrm{~cm}$ 


\section{Fundamental Constants:}

Planck's Constant: $\mathrm{h}=6.6261 \times 10^{-2 \tau} \mathrm{cm}^{2} \mathrm{~g} \mathrm{sec}^{-1}$

Speed of Light: $c=2.9979 \times 10^{10} \mathrm{~cm} \mathrm{sec}^{-1}$

Boltzmann's Constant: $\mathrm{k}_{B}=1.3807 \times 10^{-16} \mathrm{erg} \mathrm{K}^{-1}$

Newton's Constant: $\mathrm{G}=6.6720 \times 10^{-8} \mathrm{~cm}^{3} \mathrm{~g}^{-1} \mathrm{sec}^{-2} \equiv \mathrm{m}_{p l}^{-2}$

Planck Energy: $\mathrm{m}_{p l} \equiv\left(\hbar c^{5} / G\right)^{1 / 2}=1.2211 \times 10^{19} \mathrm{GoV}$

Planck Mass: $\mathrm{m}_{p l} \equiv(\hbar c / G)^{1 / 2}=2.1768 \times 10^{-5} \mathrm{~g}$

Planck Time: $\mathrm{t}_{p l} \equiv\left(\hbar G / c^{5}\right)^{1 / 2}=5.3904 \times 10^{-44} \mathrm{sec}$

Planck Length: $1_{p l} \equiv\left(\hbar G / c^{3}\right)^{1 / 2}=1.6160 \times 10^{-33} \mathrm{~cm}$

Planck Density: $\rho_{p l} \equiv c^{5} / \hbar G^{2}=5.1584 \times 10^{93} \mathrm{~g} \mathrm{~cm}^{-3}$ 


\section{Cosmological Parameters:}

Hubhle Constant: $\mathrm{H}_{0}=100 \mathrm{~h} \mathrm{~km} \mathrm{sec}^{-1} \mathrm{Mpc}^{-1}=2.1332 \mathrm{~h} \times 10^{-42} \mathrm{GeV}$

Hubble Time: $\mathrm{H}_{0}^{-1}=3.0856 \times 10^{17} \mathrm{~h}^{-1} \mathrm{sec}=9.7776 \times 10^{9} \mathrm{~h}^{-1} \mathrm{yr}$.

Hublule Distance: $\mathrm{cH}_{0}^{-1}=2997.9 \mathrm{~h}^{-1} \mathrm{Mpc}=9.2503 \times 10^{27} \mathrm{~h}^{-1} \mathrm{~cm}$

Critical Density: $\rho_{c}-3 H_{0}^{2} / 8 \pi G=1.8791 \mathrm{~h}^{2} \times 10^{-29} \mathrm{~g} \mathrm{~cm}^{-3}$

$\mathrm{H}=100 \mathrm{hm} \mathrm{sec}^{-1} \mathrm{Mpc}^{-1}=1.74695 \mathrm{~h} \times 10^{-61} t_{p l}^{-1}$ 


\section{Appendix B}

\section{The Problems of the Standard Cosmological Model}

The standard cosmological model is a compelling example of the success that may be attained by applying well-understood physics to a study of the universe at large. Nonetheless, in spite of its empirical triumphs, there is a theoretical discomfort with the model, and this unease is usually articulated as a set of "problems" pertaining to the initial conditions of the universe. In this Appendix, we examine in detail the three most common issues ... the horizon, smoothness, and flatness: problems. Our purpose is to demonstrate how inflation solves these problems.

The flatness problem, wlich we shall consider first, is the necessity to precisily adjust the value of $\Omega \equiv 3 H^{2} / 8 \pi G$ in the early universe if its value at the present epo th is to still be as close to unity as observations suggest. Physically, $\Omega$ is a ratio of the actual energy density in the universe to that minimai ?ensity (referred to as the critical density) necessary to halt the expansion of the universe. The determination of $\Omega_{o}$ (i.e., the value of $\Omega$ observed at the present epoch; is a primary objective of contemporary observational cosmology. 
The horizon and smoothness problems are essentially alternative means of stating another issue - that issue being the extent to which the observable univer se is causally connected if the standard model is correct. The data show that the universe is isotropic to a very high degree. Specifically with regard to the cosmic background radiation, the recent results from the Cosmic Background Explorer (Smoot 1992) show that the residual anisotropy in the background radiation is on the order of one part in $10^{6}$ on all angular scales. The problem is that the standard cosmological model provides that only those portions of the $2.75^{\circ} \mathrm{K}$ background radiation that are today within approximately 1 angular degree of separation of each other could have been in causal contact at the time of decoupling. Therefore, the horizon problem raises the issue as to how such a high degree of isotropy could have been attained among causally-disconnected regions of the universe.

Alternatively, this issue of causal connectedness can be phrased in terms of structure formation. We shall find that at the time of decoupling, the physical size of causally-connected regions was only a few times larger than the Milky Way galaxy. The term "smoothness problem" is often used to raise the issue as to how cosmological structures many orders of magnitude larger than this could have been formed if, as the standard model provides, the background radiation is a relic, or imprint, of the scale of inhomogeneities in the matter distribution at 
the time of decoupling. (The term "smoothness problem" is occasionally used to denote what we have called the horizon problem).

In recent years, other unresolved issues with the standard model have arisen, such as those that deal with entropy and baryon production. However, the three issues discussed in this Appendix arise only from a consideration of the overall dyanamical behavior of the standard model, and they are independent of whatever other attributes from particle physics or field theory are added to the model. Therefore, we confine our discussion to these three issues, and we shall see how they are solved by incorporating an inflationary epoch into the cosmological theory prior to the commenceineni of the standard model era.

Finally, we note that these three issues have becn addressed in many piaces in the literature, and the review monographs cited in the Introduction are only a few of these. In what follows, we have attempted to condense the discussion to the essential points.

\section{THE FLATNESS PROBLEM}

We consider equation (2.10),

$$
H^{2}=\frac{8 \pi}{3 m_{p l}^{2}} \rho-\frac{k}{R^{2}}
$$

where we have used natural units so that $G=1 / m_{p l}^{2}$. The stamlard model 
provides that from the time of the initial singularity to the present epoch the Hubble parameter has never equalled zero. Therefore we divide both sides of equation (B.1) by $H^{2}$ and define the quantity $\Omega$ as

$$
\Omega \equiv \frac{\rho}{\rho_{\text {crit }}}=\frac{8 \pi}{3 m_{p l}^{2} H^{2}} \rho
$$

so that equation (B.1) yields

$$
\Omega-1=\frac{k}{R^{2} H^{2}}
$$

If $\Omega=1$ the universe i: flat $(k=0)$, if $\Omega<1$ the universe is open $(k=-1)$, and if $\Omega>1$ the universe is closed $(k=+1)$.

The encrgy content of the standard model universe is a mixture of material particles and photons in various stages of equilibrium and relative energy density. Regardless of whether the universe as a whole is matter- or radiation-dominated, the temperature of the radiative component evolves according to $T \sim 1 / R$. We will want to use this relation since $T$ is directly measurable while $R$ is not. We designate quantities evaluated at the present epoch with a subscript "o" and quantities at some earlier epoch with the subscript "i". Equation (B.3) then yivlds

$$
\frac{\Omega_{i}-1}{\Omega_{o}-1}=\left(\frac{T_{i}}{T_{o}}\right)^{2}\left(\frac{H_{o}}{H_{i}}\right)^{2}
$$

Because the radiation density evolves as $\rho_{r} \sim T^{4} \sim 1 / R^{4}$, the first term on the righthand side of equation (B.1) would dominate the second ("curvature") 
term near the initial singularity. Therefore, at some very early moment in the universe,

$$
I_{i}^{\prime 2} \approx \frac{8 \pi}{3 m_{p l}^{2}} \rho_{i}
$$

where the radiation energy density is given by

$$
\rho_{i}=\frac{8 \pi^{3}}{90} g_{* i} T_{i}^{4}
$$

and $g_{* i}$ is the number of relativistic degrees of freedom in the "cosmological fluicl."

Combining equations (B.4), (B.5), and (B.6) yields

$$
\left|\Omega_{i}-1\right| \approx\left|\Omega_{o}-1\right|\left[\frac{90 m_{p l}^{2} H_{o}^{2}}{8 \pi^{3} g_{* i} T_{o}^{2}}\right] \frac{1}{T_{i}^{2}}
$$

Cosmological observations provide values for $\Omega_{o}, H_{0}$, and $T_{0}$; the standard model of particle physics, when embedded in the standard cosmological model, yields $g_{* i}$. The remaining free parameter in equation (B.7) is the initial tomperature. We see from equation (B.7) that as we choose carlier (hotter) initial conditions in the standard model, the initial value of $\Omega$ will approach unity with higher precision.

As an example, if we let $\left|\Omega_{o}-1\right|=0.5, H_{o}=50 \mathrm{~km} \mathrm{sec} \mathrm{sec}^{-1} \mathrm{Mpc}^{-1}, T_{o}=$ $2.75^{\circ} \mathrm{K}$, and $g_{* i}=10^{2}$ (Kolb and Turner 1990), then we find

$$
\left|\Omega_{i}-1\right| \approx \frac{4.81 \times 10^{-24}}{T_{i}^{2}} G_{e V}^{2}
$$


If we then let the initial temperature correspond to that at the Planck era $\left(T_{i} \approx\right.$ $10^{19} \mathrm{GeV}$ ), then we find that

$$
\left|\Omega_{i}-1\right| \approx 4.8 \times 10^{-62} .
$$

We see that in order for $\Omega_{o}$ to still be as near unity at the present epoch as the observations suggest, its value at the Planck epoch must be very precisely adjusted to near unity. That is, the early universe must be very finely tuned to resemble a flat univcrse, and this fine-tuning to such a unique value is cause for theoretical unease. It "rould be better if the cosmological theory were more tolerant of a broader range of initial conditions, that is, if a broader range of initial conditions could yield the universe we observe today.

\section{THE HORIZON PROBLEM}

We can integrate the equation of motion (eq. [B.1]) to obtain an ex ' ession for the horizon distance $(D)$ in terms of the redshift $z$. The expression $1+z=R_{o} / R$ serves as a dynamical definition of the redshift at some earlier epoch with scale factor $R$. After several substitutions and calculations, the horizon distance is expressed as

$$
D=\frac{1}{H_{o}(1+z)} \int_{0}^{(1+z)^{-1}} \frac{d x}{\left[\left(1-\Omega_{0}\right) x^{2}+\Omega_{o} x^{4-3} \gamma\right]^{1 / 2}}
$$

where $x=(1+z)^{-1}$ and $\gamma$ is defined in equation (2.12a). 
In order to consider the horizon and flatmess problems of the standard cosmological model, it suffices to confine our attention to the matter-dominated epoch $(\gamma=1)$ that begins with decoupling $(z=1100)$ and extends to the present epoch $(z=0)$. The integration is somewhat simm "fied, and the salient issue still remains, if we assume that $\Omega_{o}=1$. Integrating equation (B.10) yields

$$
D=\frac{2}{H_{o}(1+z)^{3 / 2}}
$$

an expression that is valid for any time during the matter era. In particular, if we compare the horizon distances at the present epoch and at decoupling, we fiurl $D_{o} / D_{d e c}=3.65 \times 10^{4}$, a figure that represents the number of causally-disconnected regions now existing in the background radiation. Partitioniu? the solid angle of a sphere into this many sections yields causally-conuected square patches of the background sky of only approximately one degree on a side.

Therefore, portions of the cosmic background radiation that differ in angular separation by more than one degree are causally-disconnected, and thould not necessarily display similar physical properties. The horizon problem raises the issue as to why the background radiation is sotropic to such high precision (1 part in $10^{6}$ ) on all angular scales.

\section{THE SMOOTHNESS PROBLEM}

The horizon problem can be restated in terms of structure formation. The 
present horizon distance is approximately $\mathrm{cH}_{o}^{-1}=3000 \mathrm{Mpc}$. Using the results from the discussion above, we find that the horizon distance at decoupling was approximately $82 \mathrm{Kpc}$, or less than three diameters of the Milky Way galaxy. However, cosmological structures exist on sca'es many orders of magnitude larger than this. Within the context of the standard model, the background radiation is an imprint of the inhomogeneities in the matier distribution at the time of dicoupling. The smoothness problem raises the issue as to how structures can form on scales exceeding the scale of causal connectedness.

\section{INFLATION}

All of these issues can be resolved if there exists in the early universe an epoch of accelerated expansion in the scale factor $(\ddot{R}>0)$. Under this cosmic "inflation," a causally-connected region can expand beyond the local horizon (with $\ddot{D}=0$ ), and the horizon and smoothness problems vanish, although the structure formation issue now has other unresolved aspects. In addition, if there is sufficient inflation, then the curvature term in equation (B.1) becomes very small relative to the other terms, and the universe appears flat regardless of the actual value of the parameter $k$.

Most inflation theories address other problems as well. For instance, the standard model explains neither the origin nor the actual value of the entropy 
of the universe, nor does it account for the observed baryon density. Inflation theories, in concert with additional assumptions regarding an underlying particle physics or field theory, have attained substantial successes in providing answers to these issues.

Unfortunately, fine-tuning problems exist in all inflation modils. At loast to some extent, the acceptance of inflation as an integral part of contemporary cosmological theory has relocated the fine-tuning problems from the domain of cosmology to the domain of field theory of matter.

Therefore, even though inflation has provided tentative answers for some of the common problems of the cosmological model, the quest contiunes for a cosmological theory that is tolerant of the broadest range of initial conditions and relies upon the fewest number of finely tuned free parameters. 
Tables 


\section{Table 1}

Solutions of Equations (4.11) for Various Values of $\gamma_{p}$

\begin{tabular}{cccc}
\hline \hline & & & \\
$\gamma_{p}$ & $1.85 \times 10^{-3}$ & $1.90 \times 10^{-3}$ & $1.95 \times 10^{-3}$ \\
\hline$y$ & $1.17 \times 10^{-66}$ & $6.21 \times 10^{-65}$ & $2.68 \times 10^{-63}$ \\
$\phi_{0}$ & 34.29 & 32.90 & 31.59 \\
$\phi_{p r}$ & 33.16 & 31.79 & 30.49 \\
$\phi_{r m}$ & 6.80 & 5.4 & 4.13 \\
$\ln \beta_{p}$ & $4.60 \times 10^{3}$ & $4.36 \times 10^{3}$ & $4.13 \times 10^{3}$ \\
$\beta_{r}$ & $5.48 \times 10^{-7}$ & $1.00 \times 10^{-5}$ & $1.58 \times 10^{-1}$ \\
$\beta_{m}$ & 1.00 & 1.00 & 1.00 \\
$C_{p}$ & $3.66 \times 10^{5}$ & $2.57 \times 10^{5}$ & $1.83 \times 10^{5}$ \\
$C_{r}$ & $1.00 \times 10^{-120}$ & $8.42 \times 10^{-120}$ & $6.34 \times 10^{-119}$ \\
$C_{m}$ & $1.27 \times 10^{-141}$ & $1.88 \times 10^{-136}$ & $1.52 \times 10^{-131}$ \\
$V_{\min }$ & $8.11 \times 10^{-140}$ & $1.21 \times 10^{-134}$ & $9.72 \times 10^{-130}$ \\
\hline
\end{tabular}


Table 1 - Continued

Solutions of Equations (4.11)

for Various Values of $\gamma_{p}$

\begin{tabular}{cccc}
\hline$\gamma_{p}$ & $2.00 \times 10^{-3}$ & $2.01 \times 10^{-3}$ & $2.0153 \times 10^{-3}$ \\
\hline$y$ & $9.61 \times 10^{-62}$ & $1.92 \times 10^{-61}$ & $2.77 \times 10^{-61}$ \\
$\phi_{0}$ & 30.34 & 30.10 & 29.97 \\
$\phi_{p r}$ & 29.25 & 29.01 & 28.88 \\
$\phi_{r m}$ & 2.90 & 2.66 & 2.53 \\
$\ln \beta_{p}$ & $3.92 \times 10^{3}$ & $3.87 \times 10^{3}$ & $3.85 \times 10^{3}$ \\
$\beta_{r}$ & $2.17 \times 10^{-3}$ & $3.60 \times 10^{-3}$ & $4.71 \times 10^{-3}$ \\
$\beta_{m}$ & 1.00 & 1.00 & 1.00 \\
$C_{p}$ & $1.31 \times 10^{5}$ & $1.23 \times 10^{5}$ & $1.19 \times 10^{5}$ \\
$C_{r}$ & $4.31 \times 10^{-118}$ & $6.25 \times 10^{-118}$ & $7.59 \times 10^{-118}$ \\
$C_{m}^{\gamma}$ & $6.96 \times 10^{-127}$ & $5.59 \times 10^{-126}$ & $1.67 \times 10^{-125}$ \\
$V_{\min }$ & $4.46 \times 10^{-125}$ & $3.58 \times 10^{-124}$ & $1.07 \times 10^{-123}$ \\
\hline
\end{tabular}


Table 2

Results for $\gamma_{p}=1.85 \times 10^{-3}$

\begin{tabular}{|c|c|c|c|c|}
\hline & $t$ & $\phi\left(m_{p l}\right)$ & $R(\mathrm{~cm})$ & $\rho\left(g m / \mathrm{cm}^{3}\right)$ \\
\hline & 0 & 34.29 & $5.58 \times 10^{-34}$ & $5.16 \times 10^{93}$ \\
\hline$\left(t_{p}\right)$ & $1.42 \times 10^{-42} \mathrm{sec}$ & 33.16 & $3.18 \times 10^{-1}$ & $3.39 \times 10^{9.3}$ \\
\hline \multirow[t]{7}{*}{$\left(t_{r}\right)$} & $8.69 \times 10^{5} y r$ & 6.80 & $1.55 \times 1^{2} \cdot 8$ & $5.95 \times 10^{-22}$ \\
\hline & $1.50 \times 10^{10} y r$ & 4.62 & $8.56 \times 10^{30}$ & $3.55 \times 10^{-30}$ \\
\hline & $1.60 \times 10^{10} y r$ & 4.61 & $8.94 \times 10^{30}$ & $3.12 \times 10^{-30}$ \\
\hline & $1.70 \times 10^{10} y r$ & 4.59 & $9.31 \times 10^{30}$ & $2.76 \times 10^{-30}$ \\
\hline & $1.80 \times 10^{10} y r$ & 4.58 & $9.67 \times 10^{30}$ & $2.16 \times 10^{-30}$ \\
\hline & $1.90 \times 10^{10} y r$ & 4.57 & $i .00 \times 10^{31}$ & $2.21 \times 10^{-30}$ \\
\hline & $2.00 \times 10^{10} y r$ & 4.56 & $1.04 \times 10^{31}$ & $2.00 \times 10^{-30}$ \\
\hline$\left(t_{m}\right)$ & $2.30 \times 10^{18} y r$ & 0.00 & $1.39 \times 10^{36}$ & $8.37 \times 10^{-115}$ \\
\hline
\end{tabular}


Table 3

Results for $\gamma_{p}=1.90 \times 10^{-3}$

\begin{tabular}{cccc}
\hline$t$ & $\phi\left(m_{p l}\right)$ & $R(\mathrm{~cm})$ & $\rho\left(\mathrm{gm} / \mathrm{cm}^{3}\right)$ \\
\hline 0 & 32.90 & $5.58 \times 10^{-34}$ & $5.16 \times 10^{93}$ \\
$\left(t_{p}\right) 1.38 \times 10^{-42} \mathrm{sec}$ & 31.79 & $4.37 \times 10^{-2}$ & $3.39 \times 10^{93}$ \\
$\left(t_{r}\right) \quad 8.69 \times 10^{5} \mathrm{yr}$ & 5.43 & $2.13 \times 10^{27}$ & $5.95 \times 10^{-22}$ \\
$1.50 \times 10^{10} \mathrm{yr}$ & 3.25 & $1.18 \times 10^{30}$ & $3.55 \times 10^{-30}$ \\
$1.60 \times 10^{10} \mathrm{yr}$ & 3.24 & $1.23 \times 10^{30}$ & $3.12 \times 10^{-30}$ \\
$1.70 \times 10^{10} \mathrm{yr}$ & 3.22 & $1.28 \times 10^{30}$ & $2.76 \times 10^{-30}$ \\
$1.80 \times 10^{10} \mathrm{yr}$ & 3.21 & $1.33 \times 10^{30}$ & $2.46 \times 10^{-30}$ \\
$1.90 \times 10^{10} \mathrm{yr}$ & 3.20 & $1.38 \times 10^{30}$ & $2.21 \times 10^{-30}$ \\
$2.00 \times 10^{10} \mathrm{yr}$ & 3.19 & $1.43 \times 10^{30}$ & $2.00 \times 10^{-30}$ \\
& & & \\
$\left(t_{m}\right) 5.97 \times 10^{15} \mathrm{yr}$ & 0.00 & $3.60 \times 10^{33}$ & $1.24 \times 10^{-40}$ \\
\hline
\end{tabular}


Table \&

Results for $\gamma_{p}=1.95 \times 10^{-3}$

\begin{tabular}{cccc}
\hline \hline & $\phi\left(m_{p l}\right)$ & $R(\mathrm{~cm})$ & $\rho\left(\mathrm{gm} / \mathrm{cm}^{3}\right)$ \\
\hline 0 & 31.59 & $5.58 \times 10^{-34}$ & $5.16 \times 10^{939}$ \\
$\left(t_{p}\right) \quad 1.34 \times 10^{-42} \mathrm{sec}$ & 30.49 & $6.64 \times 10^{-3}$ & $3.39 \times 10^{933}$ \\
$\left(t_{r}\right) \quad 8.69 \times 10^{5} \mathrm{yr}$ & 4.13 & $3.25 \times 10^{26}$ & $5.95 \times 10^{-22}$ \\
$1.50 \times 10^{10} \mathrm{yr}$ & 1.95 & $1.79 \times 10^{29}$ & $3.58 \times 10^{-30}$ \\
$1.60 \times 10^{10} \mathrm{yr}$ & 1.93 & $1.86 \times 10^{29}$ & $3.15 \times 10^{-30}$ \\
$1.70 \times 10^{10} \mathrm{yr}$ & 1.92 & $1.94 \times 10^{29}$ & $2.79 \times 10^{-30}$ \\
$1.80 \times 10^{10} \mathrm{yr}$ & 1.91 & $2.02 \times 10^{29}$ & $2.49 \times 10^{-30}$ \\
$1.90 \times 10^{10} \mathrm{yr}$ & 1.89 & $2.09 \times 10^{29}$ & $2.23 \times 10^{-30}$ \\
$2.00 \times 10^{10} \mathrm{yr}$ & 1.88 & $2.16 \times 10^{29}$ & $2.02 \times 10^{-30}$ \\
$\left(t_{m}\right) \quad 2.10 \times 10^{13} \mathrm{yr}$ & 0.00 & $1.27 \times 10^{31}$ & $1.00 \times 10^{-35}$ \\
\hline
\end{tabular}


Table 5

Results for $\gamma_{p}=2.00 \times 10^{-3}$

\begin{tabular}{cccc}
\hline$t$ & $\phi\left(m_{p l}\right)$ & $R(\mathrm{~cm})$ & $\rho\left(\mathrm{gm} / \mathrm{cm}^{3}\right)$ \\
\hline 0 & 3034 & $5.58 \times 10^{-34}$ & $5.16 \times 10^{93}$ \\
$\left(t_{p}\right) 1.31 \times 10^{-42} \mathrm{sec}$ & 29.25 & $1.11 \times 10^{-3}$ & $3.39 \times 10^{93}$ \\
$\left(t_{r}\right) \quad 8.69 \times 10^{5} \mathrm{yr}$ & 2.90 & $5.43 \times 10^{25}$ & $5.95 \times 10^{-22}$ \\
$1.50 \times 10^{10} \mathrm{yr}$ & 0.66 & $2.68 \times 10^{28}$ & $4.96 \times 10^{-30}$ \\
$1.60 \times 10^{10} \mathrm{yr}$ & 0.64 & $2.78 \times 10^{28}$ & $4.44 \times 10^{-30}$ \\
$1.70 \times 10^{10} \mathrm{yr}$ & 0.62 & $2.88 \times 10^{28}$ & $4.00 \times 10^{-30}$ \\
$1.80 \times 10^{10} \mathrm{yr}$ & 0.61 & $2.97 \times 10^{28}$ & $3.63 \times 10^{-30}$ \\
$1.90 \times 10^{10} \mathrm{yr}$ & 0.59 & $3.06 \times 10^{28}$ & $3.31 \times 10^{-30}$ \\
$2.00 \times 10^{10} \mathrm{yr}$ & 0.58 & $3.15 \times 10^{28}$ & $3.03 \times 10^{-30}$ \\
& & & \\
$\left(t_{m}\right) 9.82 \times 10^{10} \mathrm{yr}$ & 0.00 & $5.91 \times 10^{28}$ & $4.60 \times 10^{-31}$ \\
\hline
\end{tabular}


Table 6

Results for $\gamma_{p}=2.01 \times 10^{-3}$

\begin{tabular}{|c|c|c|c|c|}
\hline & $t$ & $\phi\left(m_{p l}\right)$ & $R(\mathrm{~cm})$ & $p\left(g m / \mathrm{cm}^{3}\right)$ \\
\hline & 0 & 30.10 & $5.58 \times 10^{-3.1}$ & $5.16 \times 10^{9.3}$ \\
\hline$\left(t_{p}\right)$ & $1.30 \times 10^{-42} \mathrm{sec}$ & 29.01 & $7.85 \times 10^{-.1}$ & $3.39 \times 10^{93}$ \\
\hline \multirow[t]{7}{*}{$\left(t_{r}\right)$} & $8.69 \times 10^{5} y r$ & 2.66 & $3.83 \times 10^{25}$ & $5.95 \times 10^{-22}$ \\
\hline & $1.50 \times 10^{10} y r$ & 0.35 & $1.6 .1 \times 10^{28}$ & $7.58 \times 10^{-3.30}$ \\
\hline & $1.60 \times 10^{10} y r$ & 0.32 & $1.69 \because 10^{28}$ & $6.97 \times 10^{-30}$ \\
\hline & $1.70 \times 10^{10} y r$ & 0.30 & $1.7: 3 \times 10^{28}$ & $6.15 \times 10 \times 10^{\cdots}$ \\
\hline & $1.80 \times 10^{10} y r$ & 0.28 & $1.77 \times 10^{28}$ & $6 .()^{2} 2 \times 10^{3}$ \\
\hline & $1.90 \times 10^{10} y r$ & 0.26 & $1.81 \times 10^{28}$ & $5.62 \times 10^{-30}$ \\
\hline & $2.00 \times 10^{10} y r$ & $0.2_{4}$ & $1.85 \times 10^{28}$ & $5.32 \times 10^{311}$ \\
\hline$\left(t_{m}\right)$ & $3.47 \times 10^{10} y r$ & 0.00 & $2.09 \times 10^{28}$ & $3.69 \times 103$ \\
\hline
\end{tabular}


Table 7

Results for $\gamma_{p}=2.0153 \times 10^{-3}$

\begin{tabular}{|c|c|c|c|c|}
\hline & $t$ & $\phi\left(m_{p l}\right)$ & $R(\mathrm{~cm})$ & $\rho\left(\mathrm{gm} / \mathrm{cm}^{3}\right)$ \\
\hline & 0 & 29.97 & $5.58 \times 10^{-34}$ & $5.16 \times 10^{93}$ \\
\hline$\left(t_{p}\right)$ & $1.30 \times 10^{-42} \mathrm{sec}$ & 28.88 & $6.54 \times 10^{-4}$ & $3.39 \times 10^{93}$ \\
\hline \multirow[t]{6}{*}{$\left(t_{r}\right)$} & $8.69 \times 10^{5} y r$ & 2.53 & $3.19 \times 10^{25}$ & $5.95 \times 10^{-32}$ \\
\hline & $1.50 \times 10^{10} y r$ & 0.14 & $1.16 \times 10^{28}$ & $1.24 \times 10^{-29}$ \\
\hline & $1.60 \times 10^{10} y r$ & 0.11 & $1.18 \times 10^{28}$ & $1.19 \times 10^{-29}$ \\
\hline & $1.70 \times 10^{10} y r$ & 0.08 & $1.19 \times 10^{28}$ & $1.15 \times 10^{-29}$ \\
\hline & $1.50 \times 10^{10} y r$ & 0.06 & $1.20 \times 10^{28}$ & $1.13 \times 10^{-29}$ \\
\hline & $1.90 \times 10^{10} \mathrm{yr}$ & 0.03 & $1.21 \times 10^{2.8}$ & $1.11 \times 10^{-29}$ \\
\hline$\left(t_{m}\right)$ & $2.00 \times 10^{10} y r$ & 0.00 & $1.21 \times 10^{28}$ & $1.10 \times 10^{-29}$ \\
\hline
\end{tabular}


Table 8

Summary of Results for Various Values of $\gamma_{p}$

\begin{tabular}{ccccc}
\hline$\gamma_{p}$ & $\begin{array}{c}T_{\text {initial }} \\
\left({ }^{\circ} \mathrm{K}^{\prime}\right)\end{array}$ & $\begin{array}{c}\mathrm{H}_{0} \\
\left(\mathrm{~km} \mathrm{sec} \mathrm{Mpc}^{-1}\right)\end{array}$ & $\begin{array}{c}t_{m} \\
(\text { years })\end{array}$ & $\begin{array}{c}R_{\text {max }} \\
(\mathrm{cm})\end{array}$ \\
\hline $1.85 \times 10^{-3}$ & $1.17 \times 10^{-66}$ & $32.6-43.5$ & $2.30 \times 10^{18}$ & $1.39 \times 10^{36}$ \\
$1.90 \times 10^{-3}$ & $4.50 \times 10^{-64}$ & $32.6-43.4$ & $5.97 \times 10^{15}$ & $3.60 \times 10^{33}$ \\
$1.95 \times 10^{-3}$ & $1.28 \times 10^{-61}$ & $32.5-43.3$ & $2.10 \times 10^{13}$ & $1.27 \times 10^{31}$ \\
$2.00 \times 10^{-3}$ & $2.74 \times 10^{-59}$ & $27.4-38.9$ & $9.82 \times 10^{10}$ & $5.91 \times 10^{28}$ \\
$2.01 \times 10^{-3}$ & $7.16 \times 10^{-59}$ & $18.0-29.3$ & $3.47 \times 10^{10}$ & $2.09 \times 10^{28}$ \\
$2.0153 \times 10^{-3}$ & $1.34 \times 10^{-58}$ & $0.0-16.1$ & $2.00 \times 10^{10}$ & $1.21 \times 10^{28}$ \\
$2.02 \times 10^{-3}$ & $2.17 \times 10^{-58}$ & $n a$ & $1.24 \times 10^{10}$ & $7.45 \times 10^{27}$ \\
$2.03 \times 10^{-3}$ & $6.04 \times 10^{-58}$ & $n a$ & $4.45 \times 10^{9}$ & $2.68 \times 10^{27}$ \\
\hline
\end{tabular}

